\title{
LOS SUFIJOS FORMADORES DE ADJETIVOS EN ESPAÑOL MODERNO: VALORES GENÉRICOS Y VALORES ESPECÍFICOS
}

Uno de los primeros problemas que se plantean al investigador interesado en la formación de palabras en español y, particularmente en la formación de palabras por sufijación, es la identificación de los elementos que pueden funcionar como sufijos en esta lengua y la descripción de su comportamiento, problemas a los que la bibliografía sobre el tema no ofrece una verdadera solución. En efecto, la consulta de dicha bibliografía permite constatar que la sufijación en español no ha sido objeto más que de pocos estudios sistemáticos que den cuenta, en sincronía, del conjunto del fenómeno ni desde el punto de vista descriptivo (semántico y formal) ni desde el punto de vista comparativo y sistemático (relaciones que se establecen entre los diversos sufijos). La mayor parte de las informaciones relativas a la sufijación en español se encuentran en los trabajos de carácter general y en las gramáticas del español. Estas informaciones se limitan, a menudo, a una simple enumeración de los elementos formadores, y sólo pocas veces se encuentran también datos sobre el uso de dichos elementos; las descripciones más elaboradas aparecen, normalmente, en los estudios de carácter histórico, en los que la sufijación se plantea desde el punto de vista diacrónico (Quesada 1935, García de Diego 1951, Hanssen 1966, Menéndez Pidal 1962, Lapesa 1980, Lathrop 1984, etc.). En lo que a trabajos más especializados se refiere, hay muchísimos artículos, y algunos libros, que tratan de ciertos sufijos, de un sufijo en particular $y$ a veces hasta de una sola palabra o de un par de palabras sufijadas (siempre, evidentemente, según métodos y enfoques diferentes); hay sin embargo pocos trabajos que, como el tratado de Alemany Bolufer (1920), intenten efectuar una descripción global y sistemática de todos los sufijos del español (el trabajo de Alemany Bolufer privilegia, como es normal en la época, el pun- 
to de vista diacrónico). Nuestro propósito a largo plazo es pues llegar a realizar una nueva edición del tratado de Alemany Bolufer, que describa el funcionamiento de cada uno de los sufijos de la lengua española desde una perspectiva sincrónica, aprovechando los métodos de una lingüística más moderna y tratando de establecer el sistema de relaciones que pueden ser determinadas para el conjunto de dichos sufijos.

Con este objetivo en mente, acabamos de terminar un proyecto de investigación que, basado en la observación de las lexías adjetivas sufijadas que figuran en los dieciséis diccionarios y léxicos diferentes del español que constituyen las fuentes lexicográficas del Diccionario inverso y análisis estadístico de la lengua española (Faitelson-Weiser 1988) ${ }^{1}$, trata del comportamiento general (fonológico y semántico) de los sufijos formadores de adjetivos en esta lengua? ${ }^{2}$. En el marco de dicho proyecto identificamos 87 formas sufijales ${ }^{3}$ que dan lugar a 125 sufijos formadores de adjetivos en español moderno y que se presentan bajo la forma de 129 realizaciones sufijales ${ }^{4}$ diferentes. En el presente artículo expondremos el modelo de análisis semántico que nos ha permitido identificar dichos sufijos y establecer sus valores genéricos y específicos ${ }^{5}$.

${ }^{1}$ Estas fuentes lexicográficas figuran, precedidas de un asterisco, en la sección de referencias bibliográficas.

${ }^{2}$ Este proyecto de investigación ha sido realizado gracias al generoso apoyo del Consejo de Investigaciones en Ciencias Humanas del Canadá $(C R S H)$ y del Fondo Concertado de Investigación de Quebec (FCAR), organismos a los que una vez más expresamos nuestro profundo reconocimiento.

${ }^{3}$ La forma sufijal es una representación abstracta que comprende el conjunto de los fonemas que constituyen el sufijo eventual, la indicación de sus requisitos desinenciales y la indicación del campo morfológico en el que deben inscribirse los sufijados en cuya formación participa.

${ }^{4}$ La realización sufijal es la forma concreta bajo la cual se presenta una forma sufijal o un sufijo.

${ }^{5}$ Las gramáticas del español, cuando se interesan suficientemente en estos sufijos, suelen clasificarlos de diversas maneras, sin diferir radicalmente entre ellas, pero sin coincidir tampoco. Así, por ejemplo, la gramática de R. SECo (1962, p. 125) cita tres tipos principales de sufijos formadores de adjetivos: "sufijos que expresan posesión de las cualidades contenidas en el primitivo", "sufijos gentilicios, que indican el lugar de origen", "sufijos aumentativos, despectivos y diminutivos" y habla de otros sufijos, que indican: "lo que pertenece a alguna cosa o hace relación a ella" o "capacidad o aptitud para alguna cosa". M. SECO, cuya Gramática esencial ofrece la lista más completa que hayamos visto de los sufijos del español, clasifica los sufijos formadores de adjetivos en dos grandes grupos (los apreciativos y los significativos) y atribuye a los significativos cuatro valores diferentes: "relativo a" (-al, -ar, -an-, -ian-, -ari-, -ativ-, -itiv-, -atori-, -etori-, -itori-, -ense, -eñ-, -er-, -és, -esc-, -í, -ic-, 


\section{Paráfrasis, Valores específicos y Valores genéricos}

El análisis semántico al que fueron sometidas todas las lexías de nuestro corpus tenía como objetivos distinguir los sufijados de los no sufijados y describir el comportamiento semántico de los sufijos identificados. Calificamos de sufijada toda lexía del corpus cuya definición pudo ser reducida a una paráfrasis definitoria ${ }^{6}$ que dicha lexía comparte con otras que presentan la misma terminación; el hecho de que varios adjetivos que terminan de la misma manera compartieran la misma paráfrasis definitoria, nos permitió concluir que se trataba de un posible campo morfosemántico ${ }^{7} \mathrm{y}$, consecuentemente, de un sufijo. En efecto, considerando que un sufijo es un elemento formador morfolexical y que, como tal, una de sus funciones es precisamente la atribución de un cierto grado de generalización a la lexía (Faitelson-Weiser 1980, pp. 15-26), concluimos que, si nuestras lexías podían ser inscritas en un campo morfosemántico dado, esto se debía al estatuto sufijal de la terminación. Así, después de haber verificado en las fuentes del $D I A S L E$ (y a veces también en otros trabajos lexicográficos) las definiciones de cada una de las lexías del corpus, y después de haber reformulado estas definiciones en términos de paráfrasis definitorias, identificamos los adjetivos sufijados del corpus.

Estas paráfrasis definitorias se fueron creando a medida que

-il, -in-, -ista, -os- y -un-); "que posee una cosa o tiene semejanza con ella" (-ad-, -ient-, -iz-, -on- y -ud-); "que hace la acción"' (-adiz-, -ediz-, -idiz-, -ador, -edor, -idor, -ante, -ente, -iente, -on- y -os-) y "que puede sufrir la acción"' (-able, -ible, -ader-, -eder- e -ider-).

${ }^{6}$ Una paráfrasis definitoria es una fórmula que contiene un número reducido de palabras ("Que hace [mucho]", "Que tiene [mucho]", "Que es originario [o residente] de", "Que se parece a", etc.) y un elemento que designa la clase gramatical de la noción de referencia del sufijado (S, V, Adj., Adv. Num, NPr.); basada en la definición del diccionario, la paráfrasis reduce esta última a su expresión mínima.

${ }^{7}$ El campo morfosemántico es un campo formado por un conjunto de lexías sufijadas que, perteneciendo al mismo campo morfológico - misma terminación, misma categorización y subeategorización gramaticales-, pueden ser definidas mediante una sola paráfrasis genérica. Se inscribirán pues en un mismo campo morfosemántico todas las lexías que, dentro de un campo morfológico, comparten el mismo valor genérico. Será importante, más adelante, distinguir, dentro de los sufijados, los sufijados derivados, es decir aquellos sufijados cuyo modo de formación es tal que permite identificar las diferentes fases del proceso derivacional y determinar pues el modo de formación. Esto presupone, evidentemente, un cierto parecido formal entre la lexía de referencia, que funciona entonces como lexía de base, y el sufijado. 
las íbamos necesitando para dar cuenta adecuadamente de la significación particular de cada lexía, considerando siempre la categoría gramatical de su noción de referencia; en estas condiciones utilizamos, para el conjunto de los 10524 sufijados estudiados —de los cuales 8946 son sufijados derivados-, cuarenta y siete paráfrasis definitorias distintas. Doce de ellas, sin embargo, fueron desechadas ya que no se aplicaban a un número suficiente de sufijados derivados (ver cuadro 1B); así, el número de paráfrasis definitorias conservadas pasó a treinta y cinco, como se ve en el cuadro $1 \mathrm{~A}$.

\section{Guadro $1 \mathrm{~A}$}

Las paráfrasis definitorias retenidas por orden decreciente de sus frecuencias de utilización en nuestro análisis

\begin{tabular}{llrll}
\hline & \multicolumn{2}{c}{$\%$ lexias sufijadas } & \\
\cline { 2 - 3 } Paráfrasis definitorias & Total & Derivadas & Ejemplos \\
\hline 1 Que se relaciona con S & $18.40 \%$ & $18.86 \%$ & abogad-il \\
2 Que V (mucho) & $16.25 \%$ & $17.89 \%$ & cogit-abund-o \\
3 Que es originario (o habitante) de NPr & $13.24 \%$ & $13.00 \%$ & mexic-an-o \\
4 Que tiene S (mucho) & $12.15 \%$ & $12.20 \%$ & gras-ient-o \\
5 Que se parece a S & $6.58 \%$ & $5.21 \%$ & begoñ-áce-o \\
6 Que es (más o menos) Adj & $4.93 \%$ & $5.00 \%$ & blanc-usc-o \\
7 Que puede (o debe) V: pasivo & $4.47 \%$ & $4.85 \%$ & as-ader-o \\
8 Que V (mucho): pasivo & $4.17 \%$ & $4.16 \%$ & rob-adiz-o \\
9 Que causa (o produce) S & $2.97 \%$ & $3.06 \%$ & expi-atori-o \\
10 Que puede (o debe) V & $2.41 \%$ & $2.60 \%$ & crec-eder-o \\
11 Que es partidario de S & $1.62 \%$ & $1.69 \%$ & islam-ita \\
12 Que tiene S (mucho): padece de & $1.60 \%$ & $1.70 \%$ & catarr-ient-o \\
13 Que se relaciona con NPr & $1.50 \%$ & $1.40 \%$ & cibel-e-o \\
14 Que pertenece a S & $1.25 \%$ & $0.16 \%$ & gregu-isc-o \\
15 Que es a la manera de S & $0.90 \%$ & $1.04 \%$ & abogad-esc-o \\
16 Que es originario (o habitante) de S & $0.83 \%$ & $0.57 \%$ & fronter-iz-o \\
17 Que V (mucho): perfectivo & $0.71 \%$ & $0.83 \%$ & le-id-o \\
18 Que ocupa el rango Num & $0.63 \%$ & $0.53 \%$ & cinqu-en-o \\
19 Que resulta de la división entre Num & $0.61 \%$ & $0.51 \%$ & cinqu-en-o \\
20 Que V (mucho): causativo & $0.53 \%$ & $0.56 \%$ & desliz-adiz-o \\
21 Que es aficionado a S & $0.48 \%$ & $0.53 \%$ & mujer-ieg-o \\
22 Que es partidario de NPr & $0.48 \%$ & $0.45 \%$ & jacob-ita \\
23 Que se ocupa de S & $0.43 \%$ & $0.48 \%$ & camp-us-o \\
24 Que está situado en S & $0.35 \%$ & $0.34 \%$ & limit-áne-o \\
25 Que es descendiente de NPr & $0.29 \%$ & $0.30 \%$ & ismael-ita \\
26 Que tiene NumS & $0.27 \%$ & $0.29 \%$ & catorc-én \\
27 Que se especializa en S & $0.23 \%$ & $0.26 \%$ & animal-ista \\
28 Que es a la manera de NPr & $0.21 \%$ & $0.25 \%$ & cervant-esc-o
\end{tabular}


29 Que destruye S

30 Que puede (o debe) causar $\mathrm{S}$

31 Donde se puede (o debe) $\mathrm{V}$

32 Que pertenece a NPr

33 Que se relaciona con Num

34 Que previene contra S

35 Que conduce S (mucho)

Total

\section{$0.20 \%$}

$0.17 \%$

$0.14 \%$

$0.13 \%$

$0.10 \%$

$0.10 \%$

$0.06 \%$

$99.39 \%$

10460
$0.20 \%$

$0.19 \%$

$0.17 \%$

$0.13 \%$

$0.09 \%$

$0.07 \%$

$0.07 \%$

$99.61 \%$

8911 arbor-icida rever-end-o despeñ-adiz-o alarcon-ian-o mil-en-o febri-fug-o calor-ifer-o

Cuadro $1 \mathrm{~B}$

Paráfrasis definitorias que, no aplicándose a un número sufịciente de sufijados derivados, fueron rechazadas

\begin{tabular}{lrrl}
\hline & \multicolumn{2}{c}{ \% lexias sufijadas } & \\
\cline { 2 - 3 } Paráfrasis definitorias & Total & Derivadas & Ejemplos \\
\hline 1 Que es descendiente de S & $0.31 \%$ & $0.09 \%$ & guaran-ític-o \\
2 Que puede (o debe) tener S & $0.07 \%$ & $0.08 \%$ & tor-iond-a \\
3 Que es (más o menos) Adv & $0.06 \%$ & $0.06 \%$ & asin-ota \\
4 Que se relaciona con Adv & $0.04 \%$ & $0.04 \%$ & cadañ-er-o \\
5 Qué se parece a NPr & $0.04 \%$ & $0.04 \%$ & pilat-un-o \\
6 Que se especializa en NPr & $0.02 \%$ & $0.02 \%$ & dant-ista \\
7 Que está situado Adv & $0.02 \%$ & $0.02 \%$ & tard-an-o \\
8 Que causa (o produce) S' sobre S & $0.01 \%$ & $0.01 \%$ & psic-ógen-o \\
9 Que se comporta Adv & $0.01 \%$ & $0.01 \%$ & adred-ist-o \\
10 Que es partidario de Adv & $0.01 \%$ & $0.01 \%$ & atiemp-os-o \\
11 Que está situado en NPr & $0.01 \%$ & $0.01 \%$ & danubi-an-o \\
12 Que causa (o produce) S' por S & $0.01 \%$ & $0.00 \%$ & fit-ógen-o \\
Total & $0.61 \%$ & $0.39 \%$ & \\
& 64 & 35 & \\
\hline
\end{tabular}

La mayor parte de estas paráfrasis se aplican a sufijados constituidos mediante diversas formas sufijales; sólo 14 de ellas se aplican en el caso de un número reducido de formas sufijales" se trata de: "Que conduce S (mucho)"' (-/'IFEr/-); "Que des-

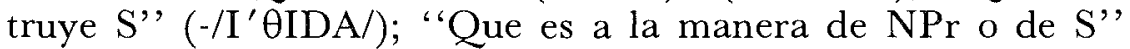
(-/'ESK/-); “Que previene contra S', (-/FUG/-); 'Que es des-

${ }^{8}$ Esto es igualmente cierto de siete de las paráfrasis rechazadas: "Que causa (o produce) S' por S" (-/'O $\chi \mathrm{EN} /-$ ); "Que causa (o produce) $\mathrm{S}^{\prime}$ sobre

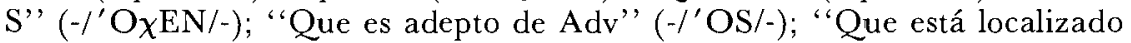
Adv" (-/'Er/-); "Que está localizado en $\mathrm{NPr}$ " (-/'AN/1-); "Que se comporta Adv" (-/'IST/-) y "Que se especializa en NPr" (-/'ISTA/). 
cendicnte de NPr" (-/'I/- e -/'ITA/); "Que pertenece a NPr" (-/'ISTA/ y $-^{\prime}$ 'AN $\left./ 1^{-9}\right)$; "Que se especializa en $\mathrm{S}$ " $\left(-/^{\prime} \mathrm{Er} /-\mathrm{e}\right.$ -/'ISTA $/)$; “Que tiene NumS'" (-/'EN/ $/{ }^{-}$y $-/$'EN/2-); "Que V (mucho): perfectivo"' (-/'AD/- e -/'ID/-); "Que ocupa el rango Num" (-/'ArJ/-, -/IM/1- y -/'EN/1-); "Que se relaciona con Num' (-/'AL/, -/'ArJ/-y -/'A $\beta /-)$; “Que resulta de la división entre Num" (-/IM/1, $\left.-/^{\prime} \mathrm{EN} /{ }_{1}-\mathrm{y}-/{ }^{\prime} \mathrm{A} \beta /-\right)$ y "Que es partidario de NPr' (-/'ES/-, -/'AN/1-, -/'ISTA/ e -l'ITA/).

En todas nuestras paráfrasis, Adj (= adjetivo), Adv (= adverbio), NPr (= nombre propio), Num (= numeral), S (= sustantivo común) y V (=verbo) representan la clase gramático-semántica de la lexía de referencia del sufijado, es decir de la noción que sirve de base referencial a la sufijación. Veintidós de dichas paráfrasis se aplican en el caso de sufijados que tienen como lexía de referencia una noción sustantiva que corresponde a la definición de un sustantivo común y nueve se aplican a nombres propios; siete fueron utilizadas en el caso de lexías de referencias verbales, tres en el caso de lexías de referencias numerales, cinco para referencias adverbiales y una para referencias adjetivas; sin embargo, al nivel de las paráfrasis retenidas, se constata una disminución en lo que a los sustantivos comunes y propios se refiere, que pasan respectivamente a dieciocho y seis, así como a las referencias adverbiales, de las que no se conserva ninguna (ver cuadro 2). Así, podemos afirmar que más de $60 \%$ de los adjetivos sufijados de nuestro corpus tienen como lexía de referencia una noción sustantiva; un poco más de una cuarta parte, una noción verbal; $5 \%$, una noción adjetiva; apenas $1 \%$, una noción numeral y una ínfima porción, una noción adverbial.

Se observa pues que en todos los casos, menos en el de la paráfrasis "Que es (más o menos) Adj", la inclusión del sufijado en un campo semántico adjetivo implica una modificación de la categoría gramatical de la referencia. Esto nos induce a dividir desde ahora nuestras paráfrasis específicas en dos grupos muy distintos uno de otro:

a) Un primer grupo constituido por la paráfrasis que acabamos de mencionar, sea “Que es (más o menos) Adj”. Esta pará-

${ }^{9}$ La cifra situada a la derecha de la segunda "/" indica que la forma sufijal en cuestión, así como el o los sufijos a los que da lugar, se encuentran en una relación de pseudo-homonimia con otra forma sufijal (por ejemplo, la representación $-^{\prime} \mathrm{EN} / 2^{-}$, utilizada para la forma sufijal de sesent-ón- $\phi / a$, indica que esta forma es diferente de la de dec-en-o/a, que corresponde a la representación $-^{\prime} \mathrm{EN} / 1^{-}$). 
Cunaro 2

Las :paráfrasis según la naturaleza 'gramático-semántica de sus lexías de referencia

\begin{tabular}{|c|c|c|c|c|c|}
\hline \multirow{2}{*}{$\begin{array}{l}\text { Lexía de } \\
\text { referencia }\end{array}$} & & \multicolumn{2}{|c|}{ \% lexias sufijadas } & \multicolumn{2}{|c|}{ Paráfrasis } \\
\hline & & total & derivadas & utilizadas & retenidas \\
\hline & comunes & $49.0 \%$ & $47.1 \%$ & 22 & 18 \\
\hline \multirow[t]{2}{*}{ Sustantivos } & propios & $15.9 \%$ & $15.6 \%$ & 9 & 6 \\
\hline & Total & $64.9 \%$ & $62.7 \%$ & 31 & 24 \\
\hline Verbos & & $28.7 \%$ & $31.1 \%$ & 7 & 7 \\
\hline Numerales & & $1.3 \%$ & $1.1 \%$ & 3 & 3 \\
\hline Adverbios & & $0.1 \%$ & $0.1 \%$ & 5 & 0 \\
\hline Adjetivos & & $4.9 \%$ & $5.0 \%$ & 1 & 1 \\
\hline \multirow{2}{*}{\multicolumn{2}{|c|}{ Total }} & $100.0 \%$ & $100.0 \%$ & 47 & 35 \\
\hline & & 10524 & 8946 & & \\
\hline
\end{tabular}

frasis corresponde bien al sentido general de todos los adjetivos que responden a ella (blancusco, negruzco, verdisco, amarillento, ricote, etc.) y en los cuales el sufijo tiene como sola función la de modificar o ponderar la intensidad del adjetivo que le sirve de referencia. Así, entre referente y derivado se establece una mera relación de inclusión vaga, parecida a la que Corbin (1987, p. 710), siguiendo a Lyons (1978, §9.4), llama una relación de hiponimia. Este comportamiento sufijal corresponde también parcialmente a lo que M. Seco (1972, pp. 186-188), Criado de Val (1958, p. 44), Marcos Marín (1972, pp. 262-264) y otros autores identifican como los sufijos apreciativos. Togeby (1965, pp. 163-167), Alarcos Llorach (1969, op. 77-78) y ia R.A.E. en su última gramática (1973, pp. 165-167) los identifican como una parte de los derivativos homogéneos, mientras que Pottier (1953 y 1962, pp. 96102) los llama infijos modíficadores y nosotros los hemos llamado en trabajos anteriores los sufijos lexicalizadores no precategorizadores, que deben distinguirse de los sufijos no lexicalizadores (Faitelson-Weiser 1980 y 1981).

b) Un segundo grupo, constituido por todas las otras paráfrasis retenidas (34), que hacen intervenir una categoría gramático-semántica distinta del adjetivo. Es en estos casos en los que el sufijo desempeña plenamente la función sufijal de "trasladar" una lexía de un campo morfosemántico dado a otro. En nuestros ejemplos, los sufijos sirven efectivamente para trasladar diversas 
nociones - sustantivas (comunes y propias), verbales y numerales- al campo de los adjetivos y, particularmente, al campo de los adjetivos de relación referencial.

En efecto, desde el punto de vista sintáctico, el sufijo formador de tales adjetivos permite, antes que nada (como la preposición de que introduce un sustantivo, o el relativo que introduce un verbo), establecer un nexo entre el sustantivo que sirve de soporte (lingüístico o referencial) al adjetivo y la lexía de referencia - nominal, verbal, numeral o adverbial- en la que se basa dicho adjetivo; al mismo tiempo, semánticamente, y dentro del adjetivo mismo, el sufijo confiere a dicho adjetivo la capacidad de referir a su lexía de referencia: el -/'eng/- de friol-eng-o permite que el sustantivo frío (lexía de referencia del adjetivo) se diga de una noción sustantiva (persona friolenga) y, al mismo tiempo, indica que friol-eng-o refiere semánticamente, de una manera u otra, a su lexía de referencia frío; de la misma manera, es el $-/$ 'oide/ de ov-oide el que le permite actuar como adjetivo y establecer una relación entre su lexía de referencia, huevo, y el sustantivo del que ov-oide se dice, ya que ov-oide no puede referir más que a huevo, etcétera.

Aceptar este razonamiento y aceptar así que todos los adjetivos sufijados de este grupo son adjetivos de relación referencial, equivale a decir que todos los adjetivos sufijados, a excepción de los que se parafrasean por “Que es (más o menos) Adj”, , pueden ser parafraseados por "Que refiere a X", paráfrasis genérica de la cual todas las demás resultarían casos específicos.

Una observación más minuciosa del conjunto de estas paráfrasis permite establecer ciertas relaciones entre ellas: si intentamos atribuir a cada paráfrasis un valor específico, resulta evidente que ciertas diferencias, debidas a menudo a la naturaleza semántico-gramatical de la noción de referencia, pueden ser abolidas, de modo que varias paráfrasis pueden ser agrupadas. Así, por ejemplo, las paráfrasis aplicadas para dar cuenta de la sufijación deverbal, pueden reunirse bajo dos rúbricas principales, a saber:

a) los agentes:

"Que V (mucho)":

"Que puede (o debe) V":

pregunt-ón- $\emptyset / a \leftarrow$ preguntar;

"Que V (mucho): causativo":

creceder-o/a $\leftarrow$ crecer ;

deslizadizo/ $a_{1} \leftarrow$ deslizar

[DRAE: 'Que hace deslizar

o se desliza fácilmente"];

"Que V (mucho): perfectivo": le-id-o/a $\leftarrow$ leer; 
"Donde se puede (o debe) V": despeñ-adiz-o/a $\leftarrow$ despeñar

[DRAE: "Dícese del lugar que es a propósito para despeñarse"']; resbal-adiz-o/ $a_{2} \leftarrow$ resbalar [DRAE: 2. "Aplícase al paraje en que hay exposición a resbalar']; andadero/ $a_{1} \leftarrow$ andar $[D R A E:$ "Aplícase al sitio o terreno donde se puede andar fácilmente']; holl-ader-o/a $\leftarrow$ hollar [DRAE: "Dícese de la parte de un camino o paraje por donde ordinariamente se transita" ], etcétera.

b) los pacientes:

"Que V (mucho): pasivo": $\quad$ robad-iz-o/a $\leftarrow$ robar;

"Que puede (o debe) V:

pasivo', :

as-ader-o/a $\leftarrow$ asar.

Pero no sólo los sufijados deverbales pueden ser agrupados así. Sufijados como cogit-abund-o/a $(\leftarrow$ cogitar $)$ o traduc-tor- $\emptyset / a(\leftarrow$ traducir) no son muy diferentes, en lo que a la porción del semantismo que puede atribuirse a la intervención del sufijo se refiere, de

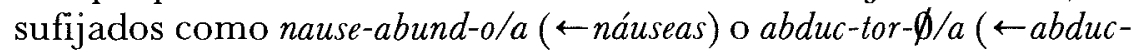
ción), aunque los dos primeros sean deverbales y los otros denominales. Así las paráfrasis "Que causa (o produce) S", "Que puede (o debe) causar S", "Que se ocupa de S", "Que es aficionado a S", "Que se especializa en S", "Que destruye S', " Que conduce S (mucho)" y "Que previene contra S", aunque dan lugar a sufijados denominales, pueden ser consideradas también como paráfrasis que representan el valor de agente, ya que en todos estos casos, como en el caso de los sufijados deverbales mencionados anteriormente, el adjetivo sufijado resultante atribuye al sustantivo del que se dice la significación general de "autor, efectivo o virtual, directo o causal, más o menos habitualmente, de una acción general o particular'". Incluimos en nuestra categoría de agente trece paráfrasis distintas, organizadas como sigue:

1) agente efectivo:

"no marcado",

causal

"Que V (mucho)";

"Que causa (o produce) S" o

"Que V (mucho): causativo".

habitual "Que se ocupa de S",

"Que es aficionado a S" o

"Que se especializa en S";

"especial",

"Que V (mucho): perfectivo",

"Que destruye S', 
"Que conduce S (mucho)" o

"Que previene contra S";

2) agente virtual:

"no marcado"

causal

"Que puede (o debe) V";

"Que puede (o debe) causar S" o

"Donde se puede (o debe) V".

El agente puede pues ser efectivo o virtual. El agente efectivo, a su vez, puede ser "no marcado", causal, habitual o "especial", y el agente virtual, "no marcado" o causal.

En el caso del agente efectivo " $n o$ marcado"', la definición del adjetivo, parafraseada por "Que V (mucho)", no contiene ninguna indicación ni restricción particular e indica sólo que el sustantivo del que el adjetivo se dice, realiza la acción verbal de manera más o menos intensa, pero suficiente para que pueda calificársele así.

El agente efectivo causal, representado por las paráfrasis "Que causa (o produce) S' o "Que V (mucho): causativo", indica que el adjetivo sufijado resultante atribuye al sustantivo del que se dice la capacidad de hacer que una acción se produzca; es una especie de agente indirecto.

Bajo la categoría de agente efectivo habitual agrupamos tres paráfrasis que indican que el sustantivo del que se dice el adjetivo sufijado tiene la habitud de hacer algo con el sustantivo que le sirve de referencia, sea porque se ocupa de él, porque es aficionado a él o porque se especializa en él.

En fin, la categoría del agente efectivo "especial", incluye las paráfrasis que tienen un sentido muy particular, ya sea porque la acción que significan ha debido ser transcrita por un verbo particular (destruye, conduce o previene contra), o porque incluye una modalidad particular (perfectivo).

En lo que al agente virtual se refiere, el agente virtual "no marcado", parafraseado por "Que puede (o debe) V' se diferencia de su homólogo efectivo sólo porque, en vez de indicar que el sustantivo del que el adjetivo se dice realiza la acción verbal de manera más o menos intensa, indica que el sustantivo del que el adjetivo se dice, puede o debe realizar la acción verbal de manera más o menos intensa.

Por último, en el caso del agente virtual causal, representado por las paráfrasis "Que puede (o debe) causar S' y "Donde se puede (o debe) V', el adjetivo sufijado resultante atribuye al sustantivo del que se dice la capacidad potencial de hacer que una 
acción se produzca; es una especie de agente indirecto potencial.

En el caso del paciente, las dos paráfrasis antes mencionadas ("Que V [mucho]: pasivo" y "Que puede [o debe] V: pasivo") son las únicas en cuyo caso el adjetivo sufijado resultante atribuye al sustantivo del que se dice la significación general de "receptor, efectivo o virtual, de la acción verbal signficada por el verbo que constituye la noción referencial de dicho sufijado"'. Serán estas dos pues las paráfrasis que incluiremos en esta categoría; en el primer caso diremos que se trata de un paciente efectivo; en el segundo, de un paciente virtual.

Las paráfrasis que acabamos de tratar se han podido reunir bajo tres valores genéricos (hiponimia, agente y paciente); veremos ahora si es posible someter las diecinueve que nos quedan a un tratamiento similar.

Tres de ellas ("Que tiene S [mucho]", "Que tiene S [mucho]: padece de" y "Que tiene NumS") han sido utilizadas en el caso de adjetivos sufijados cuya definición implica que atribuyen al sustantivo del que se dicen la posesión de uno o varios rasgos del sustantivo que sirve de base referencial al sufijado. Esta atribución se realiza de tal manera que la "posesión" más o menos intensa del rasgo en cuestión se convierte en una característica esencial o marcadora. Los adjetivos sufijados que vehiculan un tal valor han sido identificados como adjetivos de posesor y los sufijos que participan en su formación, como sufijos de posesor. La diferencia instituida entre las tres paráfrasis es mínima y reside en la noción referencial en que se basa cada sufijación. La paráfrasis "Que tiene S (mucho)" es la más general de las tres y es la que utilizamos en los casos en que la referencia nocional del sufijado era una noción sustantiva "neutra", sin ninguna connotación particular; el valor específico que le corresponde es el de posesor "no marcado".

La paráfrasis "Que tiene S (mucho): padece de" ha sido utilizada en casos en que la referencia del sufijado era un sustantivo con connotaciones negativas (catarro, piojos, etc.), de modo que el sufijado resultante vehicula más la idea de una caracterización por padecimiento que por posesión; esto nos ha llevado a hablar de un posesor negativo. Se trata más bien de una modalidad del valor anterior, que de un valor sufijal propiamente dicho.

Por último, la paráfrasis “Que tiene NumS', es una paráfrasis muy particular que se aplica a una serie reducida de adjetivos sufijados especializados y lexicalizados, en general de uso antiguo, que interiorizan en su significación el sustantivo que es mul- 
tiplicado por el numeral a la base de la sufijación: catorc-en-o, (DRAE: 2. "V . paño catorceño = Gierta especie de paño basto, cuya urdimbre consta de 14 centenares de hilos. U.t.c.s.'"), doc-en-o (DRAE: 2. "Aplícase al paño o a otro tejido de lana, cuya urdimbre consta de 12 centenares de hilos. U.t.c.s.m.'), catorc-én (DRAE: "Se dice del madero en rollo de 14 medias varas de longitud y un diámetro de 10 a 13 dedos. U.m.c.s.'), mil-en-o (DRAE: "Dícese de las telas cuya urdimbre se compone de mil hilos"), etc. Este caso fue asimilado al del posesor " no marcado", puesto que al final consideramos que se trata de simples casos de lexicalización de aquél.

De las dieciséis paráfrasis que quedan, ocho ("Que es originario [o habitante] de NPr", "Que es originario [o habitante] de S", "Que es partidario de S", "Que es partidario de NPr", "Que pertenece a S", "Que pertenece a NPr", "Que está situado en S' y "Que es descendiente de NPr') han sido utilizadas para definir adjetivos que hacen que el sustantivo del que se dicen sea identificado a la noción referencial del sufijado; esta identificación lo lleva a ser incluido en un grupo cuyos individuos tienen en común un mismo origen - geográfico ("Que es originario [o habitante] de NPr o de S') o histórico ("Que pertenece a S o a NPr') o ideológica ("Que es descendiente de NPr"')-, una misma filiación -étnica ("Que es partidario de $\mathrm{S}$ o de NPr') - o una misma ubicación ("Que está situado en S'). Esta constatación nos ha llevado a hablar, en el caso de estos sufijados, de adjetivos de identificación y a atribuir a los sufijos que intervienen en su formación dicho valor genérico. La identificación puede producirse por origen, por adhesión, por descendencia, por filiación étnica o por localización.

El valor de origen, correspondiente a las paráfrasis "Que es originario (o habitante) de NPr o de S', caracteriza adjetivos sufijados que atribuyen al sustantivo del que se dicen la propiedad de identificarse con su lugar de origen o de residencia, representado en general por un nombre propio, pero a veces también por un nombre común.

En el caso del valor de adhesión, representado por las paráfrasis "Que es partidario de S o de NPr", el adjetivo sufijado resultante atribuye al sustantivo del que se dice la propiedad de identificarse con una corriente ideológica, filosófica, etcétera.

Bajo la categoría de filiación étnica rupamos dos paráfrasis que indican que el sustantivo del que se dice el adjetivo sufijado se identifica con un grupo étnico: "Que pertenece a NPr o a S". 
La descendencia indica que el sustantivo del que se dice el adjetivo sufijado se identifica con otros por un ascendiente común; corresponde pues a la paráfrasis "Que es descendiente de NPr".

Finalmente, la localización responde a la paráfrasis "Que está situado en S',

De las ocho paráfrasis que quedan, tres han sido atribuidas a adjetivos sufijados que establecen, entre su lexía de referencia y el sustantivo del que se dicen, una relación de parecido. Se trata de-las paráfrasis "Que se parece a S', "Que es a la manera de S' y "Que es a la manera de NPr'. La primera de ellas implica un parecido físico (de forma, de color, de tamaño, etc.) entre los dos sustantivos; la segunda y la tercera, en cambio implican un parecido modal.

En fin, de las cinco paráfrasis restantes, tres ("Que se relaciona con S", "Que se relaciona con NPr' y "Que se relaciona con Num'") establecen entre el soporte del adjetivo sufijado y su noción de referencia un nexo vago de simple relación ${ }^{10}$. Las otras dos, instituyen un nexo muy particular entre el soporte del adjetivo y su referencia numeral y corresponden a las categorías tradicionalmente reconocidas como numerales ordinales ("Que ocupa el rango Num") y numerales partitivos ("Que resulta de la división entre Num"). Agrupamos estas cinco paráfrasis bajo el valor genérico de relación, y dividimos este valor genérico en varios valores específicos, como sigue: relación general, relación numeral general, relación numeral ordinal y relación numeral partitiva.

Lo que acabamos de decir, se representa en la figura 1 y se sintetiza en el cuadro 3. En este cuadro se observa que el agente es el valor genérico más frecuente de nuestro corpus, seguido por la simple relación y la identificación; viene luego el posesor, seguido por el paciente y el parecido $\mathrm{y}$, por último, la hiponimia.

${ }^{10}$ Este valor general puede constituir el valor propio de un sufijado, pero puede también, a veces, resultar, en el caso de un sufijado que tiene otro valor específico, de una especie de "neutralización" de su valor original. Si hablamos de "neutralización" es porque el fenómeno constatado recuerda, mutatis mutandis, lo que les sucede en fonología a ciertos fonemas que, bajo ciertas condiciones, pierden algunos de sus rasgos distintivos. Así, por ejemplo, los adjetivos gentilicios como canadi-ense, mexic-an-o, etc. que, según nuestra clasificación corresponderían al valor de identificación cuando se dicen de un humano, podrían con relativa facilidad, perder este valor y expresar un "simple valor de relación" en ciertos contextos, al decirse, por ejemplo, de un sustantivo no humano. 
FIGURA 1

Valores genéricos y valores específicos

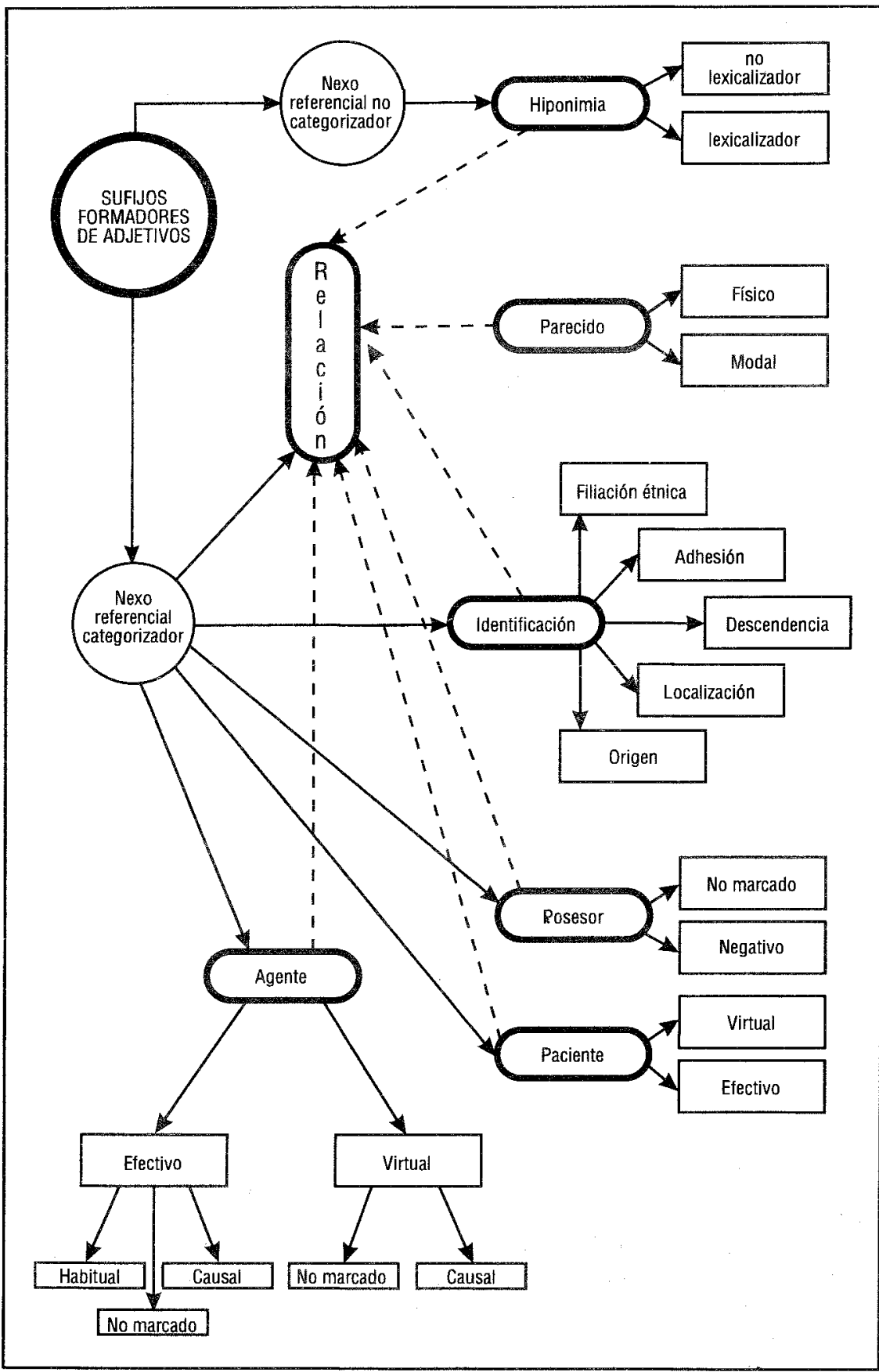


Cuadro 3

La sufijación considerada: valores $y$ paráfrasis

\begin{tabular}{|c|c|c|c|c|c|}
\hline \multirow{2}{*}{$\begin{array}{c}\text { Valores } \\
\text { genéricos }\end{array}$} & \multirow{2}{*}{\multicolumn{2}{|c|}{$\begin{array}{c}\text { Valores } \\
\text { especificos }\end{array}$}} & \multirow[b]{2}{*}{ Paráfrasis definitorias } & \multicolumn{2}{|c|}{$\%$ sufijados } \\
\hline & & & & Total & $\begin{array}{l}\text { Deri- } \\
\text { vados }\end{array}$ \\
\hline \multirow{7}{*}{$\begin{array}{l}\mathrm{A} \\
\mathrm{G} \\
\mathrm{E} \\
\mathrm{N} \\
\mathrm{T} \\
\mathrm{E}\end{array}$} & \multirow{4}{*}{$\begin{array}{l}\text { Efec- } \\
\text { tivo }\end{array}$} & & Que V (mucho) & $16.25 \%$ & $17.89 \%$ \\
\hline & & causal & $\begin{array}{l}\text { Que causa (o produce) S } \\
\text { Que V (mucho): causativo }\end{array}$ & $\begin{array}{l}2.97 \% \\
0.53 \% \\
\end{array}$ & $\begin{array}{l}3.06 \% \\
0.56 \% \\
\end{array}$ \\
\hline & & habitual & $\begin{array}{l}\text { Que se ocupa de } S \\
\text { Que es aficionado a } S \\
\text { Que se especializa en } S\end{array}$ & $\begin{array}{l}0.43 \% \\
0.48 \% \\
0.23 \%\end{array}$ & $\begin{array}{l}0.48 \% \\
0.53 \% \\
0.26 \%\end{array}$ \\
\hline & & \multirow[t]{2}{*}{ especial } & $\begin{array}{l}\text { Que V (mucho): perfectivo } \\
\text { Que destruye S } \\
\text { Que conduce S (mucho) } \\
\text { Que previene contra S }\end{array}$ & $\begin{array}{l}0.71 \% \\
0.20 \% \\
0.06 \% \\
0.10 \% \\
\end{array}$ & $\begin{array}{l}0.83 \% \\
0.20 \% \\
0.07 \% \\
0.07 \% \\
\end{array}$ \\
\hline & & & Que puede (o debe) V & $2.41 \%$ & $2.60 \%$ \\
\hline & Virtual & causal & $\begin{array}{l}\text { Que puede (o debe) causar S } \\
\text { Donde se puede (o debe) V }\end{array}$ & $\begin{array}{l}0.17 \% \\
0.14 \%\end{array}$ & $\begin{array}{l}0.19 \% \\
0.17 \%\end{array}$ \\
\hline & \multicolumn{3}{|r|}{ 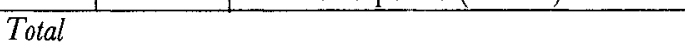 } & $24.68 \%$ & $\overline{26.91 \%}$ \\
\hline \multirow{3}{*}{$\begin{array}{l}\text { RE- } \\
\text { LA- } \\
\text { CIÓN }\end{array}$} & \multicolumn{2}{|c|}{ General } & $\begin{array}{l}\text { Que se relaciona con } \mathrm{S} \\
\text { Que se relaciona con } \mathrm{NPr}\end{array}$ & $\begin{array}{r}18.40 \% \\
1.50 \%\end{array}$ & $\begin{array}{r}18.86 \% \\
1.40 \%\end{array}$ \\
\hline & $\begin{array}{l}\text { Nume- } \\
\text { ral }\end{array}$ & $\begin{array}{l}\text { Ordinal } \\
\text { Partit. } \\
\text { Gen. }\end{array}$ & $\begin{array}{l}\text { Que ocupa el rango Num } \\
\text { Que resulta de la div. entre Num } \\
\text { Que se relaciona con Num }\end{array}$ & $\begin{array}{l}0.63 \% \\
0.61 \% \\
0.10 \%\end{array}$ & $\begin{array}{l}0.53 \% \\
0.51 \% \\
0.09 \%\end{array}$ \\
\hline & \multicolumn{3}{|l|}{ Total } & $21.24 \%$ & $21.39 \%$ \\
\hline \multirow[t]{2}{*}{$\begin{array}{l}\text { IDEN- } \\
\text { TIFI- } \\
\text { CA- } \\
\text { CIÓN }\end{array}$} & $\begin{array}{l}\text { Filiaciór } \\
\text { Descend } \\
\text { Localiza }\end{array}$ & $\begin{array}{l}\text { étnica } \\
\text { lencia } \\
\text { ción }\end{array}$ & $\begin{array}{l}\text { Que es orig. (o habit.) de NPr } \\
\text { Que es orig. (o habit.) de S } \\
\text { Que es partidario de S } \\
\text { Que es partidario de NPr } \\
\text { Que pertenece a S } \\
\text { Que pertenece a NPr } \\
\text { Que es fescendiente de NPr } \\
\text { Que está situado en S }\end{array}$ & $\begin{array}{r}13.24 \% \\
0.83 \% \\
1.62 \% \\
0.48 \% \\
1.25 \% \\
0.13 \% \\
0.29 \% \\
0.35 \% \\
\end{array}$ & $\begin{array}{r}13.00 \% \\
0.57 \% \\
1.69 \% \\
0.45 \% \\
0.16 \% \\
0.13 \% \\
0.30 \% \\
0.34 \% \\
\end{array}$ \\
\hline & \multicolumn{3}{|c|}{ Total } & $18.19 \%$ & $16.64 \%$ \\
\hline \multirow{3}{*}{$\begin{array}{l}\text { PO- } \\
\text { SE- } \\
\text { SOR }\end{array}$} & \multicolumn{2}{|c|}{ Efectivo } & $\begin{array}{l}\text { Que tiene S (mucho) } \\
\text { Que tiene NumS }\end{array}$ & $\begin{array}{r}12.15 \% \\
0.27 \%\end{array}$ & $\begin{array}{r}12.20 \% \\
0.29 \%\end{array}$ \\
\hline & & negat. & Que tiene S (mucho): padece de & $1.60 \%$ & $1.70 \%$ \\
\hline & \multicolumn{3}{|l|}{ Total } & $14.02 \%$ & $14.19 \%$ \\
\hline \multirow{2}{*}{$\begin{array}{c}\text { PA- } \\
\text { CIEN- } \\
\text { TE }\end{array}$} & \multicolumn{2}{|l|}{$\begin{array}{l}\text { Virtual } \\
\text { Efectivo }\end{array}$} & $\begin{array}{l}\text { Que puede (o debe) V: pasivo } \\
\text { Que V (mucho): pasivo }\end{array}$ & $\begin{array}{l}4.47 \% \\
4.17 \%\end{array}$ & $\begin{array}{l}4.85 \% \\
4.16 \%\end{array}$ \\
\hline & \multicolumn{3}{|l|}{ Total } & $8.64 \%$ & $9.01 \%$ \\
\hline \multirow[t]{2}{*}{$\begin{array}{l}\text { PA- } \\
\text { RECI- } \\
\text { DO }\end{array}$} & & $\begin{array}{l}\text { Que se parece a } S \\
\text { Que es a la manera de } S \\
\text { Que es a la manera de NPr }\end{array}$ & $\begin{array}{l}6.58 \% \\
0.90 \% \\
0.21 \%\end{array}$ & $\begin{array}{l}5.21 \% \\
1.04 \% \\
0.25 \%\end{array}$ \\
\hline & \multicolumn{3}{|l|}{ Total } & $7.69 \%$ & $6.50 \%$ \\
\hline \multicolumn{3}{|c|}{ HIPONIMIA } & Que es (más o menos) Adj & $4.93 \%$ & $5.00 \%$ \\
\hline Total & & \multicolumn{2}{|l|}{$\begin{array}{l}\% \\
\text { absoluto }\end{array}$} & $\begin{array}{c}100.00 \% \\
10524\end{array}$ & $\begin{array}{l}100.00 \% \\
8946\end{array}$ \\
\hline
\end{tabular}


2. VALORES GENÉRICOS Y HOMONIMIA SUFIJAL

Una vez establecidos los valores genéricos y específicos de todas nuestras lexías, procedimos a la determinación de la lista de los sufijos formadores de adjetivos en español moderno según los criterios siguientes:

1) Cuando un valor genérico caracterizaba más de $50 \%$ de las lexías sufijadas formadas mediante una misma forma sufijal y 3 o más sufijados derivados, le atribuíamos a esta forma sufijal el valor genérico en cuestión como valor principal;

2) si un valor genérico caracterizaba menos de $51 \%$ pero más de $20 \%$ de las lexías sufijadas formadas mediante una misma forma sufijal y 3 o más sufijados derivados, este valor se le atribuía a la forma sufijal en cuestión como valor concurrente;

3) cuando un valor genérico caracterizaba menos de $21 \%$ de los sufijados que comprendían una forma sufijal dada, pero más de $10 \%$ y 3 sufijados derivados o más, considerábamos este valor como un valor ocasional de la forma sufijal en cuestión;

4) en fin, cuando un valor genérico no caracterizaba más que $10 \%$ o menos de los sufijados derivados mediante una forma sufijal dada, o cuando no era vehiculado por un mínimo de 3 sufijados derivados así formados, consideramos que se trataba de for maciones idiosincráticas.

Sólo cuando la primera o la segunda de estas condiciones se encontraba satisfecha declaramos una forma sufijal apta para vehicular el valor genérico en cuestión, hecho que le confería el estatu to de sufijo dotado de un significante (la representación de la forma sufijal con sus realizaciones correspondientes) y de un significado (el valor genérico retenido). Cuando una forma sufijal vehiculaba en dichas condiciones más de un valor genérico, concluimos que daba lugar a sufijos homonímicos.

El cuadro 4 presenta nuestras 87 formas sufijales y los valores de los sufijos a los que cada una da lugar. Se observará que 55 de nuestras formas sufijales dan lugar a sufijos no homonímicos ${ }^{11}$.

${ }^{11}$ Ha de notarse sin embargo que en el caso de diez de estas formas sufijales, relativamente poco productivas en el DIASLE (hiponimia: -/'ANK/- e $-/^{\prime} \mathrm{I} /-$, menos de 50 lexías adjetivas; agente: -/'END/- e -/'IKA/, menos de 50 lexías adjetivas y $-/^{\prime} \mathrm{AN} / 2^{-}$, menos de 100 lexías adjetivas; relación: -/'ENG/-, -/'IN/ y -/AK/-, menos de 50 lexías adjetivas; posesor: $-/^{\prime} \mathrm{OT} / 2^{-}$, menos de 50 lexías adjetivas e $-/$ I $\mathrm{I} \theta /-$, menos de 100 lexías adjetivas), no pudimos retener ningún valor como valor principal, ya que el valor que vehiculan con más frecuencia compite fuertemente con diversos valores ocasionales e idiosincráticos. 
Las 32 formas sufijales restantes dan lugar a los setenta sufijos homonímicos de nuestro corpus: veintiséis de ellas dan lugar a dos sufijos cada una; treinta y cinco de estos sufijos presentan un valor concurrente; diecisiete, un valor principal. En fin, cada una de las otras seis formas sufijales (-/' $\mathrm{IN} / 2^{-},-/^{\prime} \mathrm{E} /-,-/ \mathrm{E} /-,-/^{\prime} \mathrm{Er} /-$, -/'ISTA/ y -/'ON/-) da lugar a tres sufijos diferentes.

\section{Cuadro 4}

Valores génericos vehiculados por las diversas formas sufijales

\begin{tabular}{|c|c|c|c|c|c|c|c|c|c|c|}
\hline \multicolumn{3}{|c|}{ Formas sufijales } & \multicolumn{7}{|c|}{ Valores genéricos $^{12}$} & \multirow[b]{2}{*}{$\begin{array}{l}\text { Sufi- } \\
\text { jos }\end{array}$} \\
\hline Rep & esentación & $\begin{array}{l}\text { Producti- } \\
\text { vidad }^{13}\end{array}$ & Agente & Paciente & Posesor & $\begin{array}{l}\text { Identifi- } \\
\text { cación }\end{array}$ & $\begin{array}{l}\text { Rela- } \\
\text { ción }\end{array}$ & $\begin{array}{l}\text { Pare- } \\
\text { cido }\end{array}$ & $\begin{array}{l}\text { Hipo- } \\
\text { nimia }\end{array}$ & \\
\hline 1 & $-/^{\prime} \mathrm{AK} /-$ & $x x x$ & idi. & & oc. & (1) & (2) & oc. & oc. & 0 \\
\hline 2 & $-/^{\prime} \mathrm{A} \theta \mathrm{E} /-$ & $\mathrm{xxx}$ & & & idi. & & idi. & 1 & idi. & 1 \\
\hline 3 & $-/^{\prime} \mathrm{AD} /-$ & $\mathrm{xxxxx}^{*}$ & oc. & 1 & (2) & idi. & idi. & idi. & & 2 \\
\hline 4 & $-l^{\prime} \mathrm{AJK} /-$ & $\mathrm{x}$ & & & & (2) & 1 & & & \\
\hline 5 & $-/{ }^{\prime} \mathrm{AL} /$ & $\operatorname{xxxxx} *$ & idi. & idi. & idi. & & 1 & idi. & idi. & \\
\hline 6 & $-I^{\prime} \mathrm{AN} / 1^{-}$ & $\mathrm{xxxxx}^{*}$ & idi. & idi. & idi. & 1 & oc. & idi. & idi. & \\
\hline 7 & $-I^{\prime} \mathrm{AN} / 2^{-}$ & $\mathrm{xx}$ & (1) & idi. & oc. & idi. & & idi. & & \\
\hline 8 & $-/{ }^{\prime} \mathrm{ANK} /-$ & $\mathrm{x}$ & & & oc. & oc. & & oc. & (1) & \\
\hline 9 & $-/ \mathrm{AND} /-$ & $x$ & oc. & 1 & & oc. & idi. & oc. & & \\
\hline 10 & $-/{ }^{\prime} \mathrm{ANE} /-$ & $\mathrm{x}$ & oc. & & oc. & 1 & oc. & oc. & idi. & \\
\hline 11 & $-/{ }^{\prime} \mathrm{ANG} /-$ & $\mathrm{x}$ & & & & idi. & idi. & idi. & 1 & \\
\hline 12 & $-I^{\prime} \mathrm{Ar} /$ & $\mathrm{xxxx}$ & idi. & idi. & idi. & idi. & 1 & oc. & idi. & \\
\hline 13 & $-/{ }^{\prime} \mathrm{ArJ} /-$ & $\mathrm{xxxx}^{*}$ & oc. & & (2) & idi. & (1) & idi. & idi. & \\
\hline 14 & $-I^{\prime} \mathrm{A} \beta /-$ & $\mathrm{xx}$ & & & & idi. & 1 & & idi. & \\
\hline 15 & $-/{ }^{\prime} \mathrm{A} \theta /-$ & $\mathrm{x}$ & & & (2) & & & & 1 & \\
\hline 16 & $-/ \mathrm{E} /-$ & $\mathrm{xxx}$ & idi. & & idi. & (1) & (2) & (3) & oc. & \\
\hline 17 & $-\gamma^{\prime} \mathrm{EK} /-$ & $\mathrm{xxx}$ & idi. & idi. & oc. & 1 & idi. & idi. & idi. & \\
\hline 18 & $-/^{\prime} \mathrm{EKA} /$ & $\mathrm{x}$ & & & oc. & 1 & & & & \\
\hline 19 & $/{ }^{\prime} \mathrm{EG} /-$ & $x x$ & oc. & & idi. & (2) & 1 & idi. & idi. & \\
\hline 20 & $-/^{\prime} \mathrm{E} \chi /-$ & $\mathrm{x}$ & & idi. & (2) & oc. & idi. & idi. & (1) & \\
\hline 21 & $-/ \mathrm{EN} / 1^{-}$ & $x x x$ & & & (2) & oc. & 1 & idi. & idi. & \\
\hline 22 & $-/^{\prime} \mathrm{EN} / 2^{-}$ & $\mathrm{x}$ & & & 1 & oc. & & & & \\
\hline 23 & $-/{ }^{\prime} \mathrm{ENK} /-$ & $\mathrm{x}$ & idi. & & idi. & oc. & oc. & & 1 & \\
\hline
\end{tabular}

12 Las cifras en estas columnas indican el rango de los valores específicos retenidos en el caso de cada sufijo; entre ( ), significan que se trata de un valor concurrente. "oc." significa un valor ocasional e "idi.", un valor idiosincrático.

${ }^{13} \mathrm{xxx \times x}=$ más 500 lexías adjetivas en el DIASLE; $\mathrm{xxxx}=$ entre $250 \mathrm{y}$ 499 lexías adjetivas; $\mathrm{xxx}=$ entre 100 y 249 lexías adjetivas; $\mathrm{xx}=$ entre 50 y 99 lexías adjetivas; $x=$ menos de 50 lexías adjetivas. Un asterisco situado después de un conjunto de " $x$ " indica que el sufijo ha sido estudiado a partir de un muestreo. 
Cuadro 4 (continúa)

\begin{tabular}{|c|c|c|c|c|c|c|c|c|c|c|}
\hline \multicolumn{3}{|c|}{ Formas sufijales } & \multicolumn{7}{|c|}{ Valores genéricos } & \multirow[b]{2}{*}{$\begin{array}{c}\text { Sufi- } \\
\text { jos }\end{array}$} \\
\hline \multicolumn{2}{|c|}{ Representación } & $\begin{array}{l}\text { Producti- } \\
\text { vidad }\end{array}$ & Agente & Paciente & Posesor & $\begin{array}{l}\text { Identifi- } \\
\text { cación }\end{array}$ & $\begin{array}{l}\text { Rela- } \\
\text { ción }\end{array}$ & $\begin{array}{c}\text { Pare- } \\
\text { cido }\end{array}$ & $\begin{array}{l}\text { Hipo- } \\
\text { nimia }\end{array}$ & \\
\hline 24 & $-/$ END/. & $\mathrm{x}$ & (1) & idi. & idi. & & idi. & idi. & & 1 \\
\hline 25 & -/'ENG/- & $\mathrm{x}$ & & & idi. & & (1) & oc. & idi. & 1 \\
\hline 26 & -/'ENSE/ & $\mathrm{xxxxx}^{*}$ & & idi. & & 1 & & & & 1 \\
\hline 27 & $-/{ }^{\prime} \mathrm{ENT} /-$ & $\mathrm{xxx}$ & oc. & & 1 & idi. & idi. & idi. & & 1 \\
\hline 2 & $-/^{\prime} \mathrm{EJI}_{\mathrm{I}} /-$ & $x \times x x x^{*}$ & idi. & & idi. & 1 & idi. & idi. & & 1 \\
\hline 29 & $-/$ Er/- & $\mathrm{xxxxx}^{*}$ & (1) & & idi. & (2) & (3) & idi. & idi. & 3 \\
\hline 30 & $-l^{\prime} \mathrm{ES} /-$ & $x x x$ & & & & 1 & idi. & idi. & & 1 \\
\hline 31 & -/'ESK/- & $\mathrm{xxx}$ & idi. & & idi. & idi. & oc. & 1 & idi. & 1 \\
\hline 32 & /'ETE/ & $\mathrm{x}$ & (1) & & & oc. & & idi. & (2) & 2 \\
\hline 33 & $-/ ' \mathrm{I} /$ & $x x x$ & & & oc. & 1 & oc. & idi. & & 1 \\
\hline 3 & $-I^{\prime} \mathrm{I} /-$ & $\mathrm{x}$ & oc. & oc. & oc. & oc. & idi. & idi. & (1) & 1 \\
\hline $3 !$ & $-/$ IKA / & $\mathrm{x}$ & (1) & & idi. & idi. & & idi. & & 1 \\
\hline 36 & $-/^{\prime} I \theta \mathrm{J} /-$ & $x x$ & idi. & idi. & idi. & idi. & 1 & & oc. & 1 \\
\hline $3 i$ & $-/$ IKOLA $/$ & $\mathrm{x}$ & idi. & & & 1 & (2) & & & 2 \\
\hline 38 & $-/^{\prime} \mathrm{ID} /-$ & $\mathrm{xxxx}^{*}$ & (2) & (1) & idi. & idi. & idi. & & idi. & 2 \\
\hline 35 & -/'IFEr/- & $x x$ & (2) & & 1 & & & idi. & & 2 \\
\hline 40 & $-/^{\prime} \mathrm{IFIK} /-$ & $\mathrm{x}$ & (1) & & oc. & & (2) & & oc. & 2 \\
\hline 41 & $-/^{\prime} \mathrm{I} \chi \mathrm{Er} /-$ & $\mathrm{x}$ & oc. & & 1 & & & idi. & & 1 \\
\hline 42 & $-/$ IL/ & $\mathrm{xxx}$ & idi. & idi. & idi. & idi. & 1 & idi. & idi. & 1 \\
\hline 43 & $-l^{\prime} \mathrm{IN} /$ & $\mathrm{x}$ & oc. & & & idi. & (1) & & & 1. \\
\hline 44 & $/^{\prime} \mathrm{IN} / 1^{-}$ & $\mathrm{xxxx}^{*}$ & idi. & & idi. & 1 & (2) & idi. & idi. & 2 \\
\hline 4 & $-/^{\prime} \mathrm{IN} / 2^{-}$ & $\mathrm{xx}$ & (2) & & idi. & (1) & oc. & & 3) & 3 \\
\hline 46 & $-/$ INE/- & $\mathrm{x}$ & & & oc. & & oc. & 1 & idi. & 1 \\
\hline 4 & $-/^{\prime} \mathrm{J} O N D /-$ & $\mathrm{x}$ & (2) & & 1 & & & & & 2 \\
\hline 48 & -/'ISK/- & $\mathrm{x}$ & oc. & & oc. & 1 & oc. & oc. & idi. & 1 \\
\hline 49 & -/'IST/- & $x$ & 1 & & & (2) & & & & 2 \\
\hline 50 & $-f^{\prime}$ ISTA $/$ & $\mathrm{xxxxy}$ & (2) & & idi. & (1) & (3) & & & 3 \\
\hline 51 & $-/{ }^{\prime} 1 \mathrm{~T} /$ & $x x x$ & & oc. & oc. & idi. & idi. & (2) & (1) & 2 \\
\hline 52 & -ITA/ & $x x x$ & & & idi. & 1 & idi. & idi. & idi. & 1 \\
\hline 53 & $-/^{\prime} \mathrm{I} \beta /-$ & $\mathrm{xxxxx}^{*}$ & 1 & idi. & idi. & idi. & oc. & idi. & idi. & 1 \\
\hline 54 & ${ }^{\prime} \mathrm{I} \mathrm{I} \theta /$ & $x x$ & oc. & idi. & (1) & idi. & oc. & oc. & oc. & 1 \\
\hline 55 & $-/^{\prime} \mathrm{O} \chi \mathrm{EN} /-$ & $\mathrm{x}$ & 1 & & idi. & idi. & idi. & & & 1 \\
\hline 56 & -/'OJDE/ & $x$ & & & idi. & $o c$ & idi. & 1 & idi. & 1 \\
\hline 57 & $-/{ }^{\prime} \mathrm{ON} /-$ & $\operatorname{xxxxx}$ & (1) & idi. & (2) & idi. & idi. & idi. & (3) & 3 \\
\hline 58 & $-/^{\prime} \mathrm{Or} / 1^{-}$ & $x x x x x^{*}$ & 1 & & idi. & idi. & idi. & & & 1 \\
\hline 59 & $-/^{\prime} \mathrm{Or} / 2^{-}$ & $\mathrm{x}$ & 1 & & & & & & & 1 \\
\hline 60 & $-/^{\prime} \mathrm{OrJ} /-$ & $\mathrm{xxxx}$ & 1 & idi. & idi. & & (2) & idi. & idi. & 2 \\
\hline 61 & $-/{ }^{\prime} \mathrm{OS} /-$ & $\mathrm{xxxxx}^{*}$ & (2) & idi. & 1 & idi. & idi. & idi. & idi. & 2 \\
\hline 62 & $-/^{\prime O T} / 1^{-}$ & $\mathrm{x}$ & & & idi. & oc. & & idi. & 1 & 1 \\
\hline 63 & $-/^{\prime} \mathrm{OT} / 2^{-}$ & $\mathrm{x}$ & idi. & oc. & (1) & idi. & & oc. & & 1 \\
\hline 64 & -/'OTA/ & $\mathrm{x}$ & & & idi. & 1 & & idi. & & 1 \\
\hline 65 & $-/{ }^{\prime} \mathrm{UK} /-$ & $x x$ & idi. & & (2) & idi. & idi. & idi. & (1) & 2 \\
\hline 66 & $-/ ' \mathrm{UD} /-$ & $\mathrm{xxxx}^{*}$ & idi. & & 1 & idi. & idi. & oc. & idi. & 1 \\
\hline 67 & -/'WEL/. & $\mathrm{x}$ & & & & idi. & & oc. & 1 & 1 \\
\hline
\end{tabular}


Guadro 4 (concluye)

\begin{tabular}{|c|c|c|c|c|c|c|c|c|c|c|}
\hline \multicolumn{3}{|c|}{ Formas sufijales } & \multicolumn{7}{|c|}{ Valores genéricos } & \multirow[b]{2}{*}{$\begin{array}{l}\text { Suifi- } \\
\text { jos }\end{array}$} \\
\hline \multicolumn{2}{|c|}{ Representación } & \multirow{2}{*}{$\begin{array}{l}\text { Producti- } \\
\text { vidad } \\
\mathrm{xxx}\end{array}$} & \multirow{2}{*}{$\frac{\text { Agente }}{\text { idi. }}$} & \multirow[t]{2}{*}{ Paciente } & \multirow{2}{*}{$\frac{\text { Posesor }}{\text { idi. }}$} & \multirow{2}{*}{$\begin{array}{c}\begin{array}{c}\text { Identifi- } \\
\text { cación }\end{array} \\
1\end{array}$} & \multirow{2}{*}{$\frac{\begin{array}{c}\text { Rela- } \\
\text { ción }\end{array}}{(2)}$} & \multirow{2}{*}{$\begin{array}{c}\begin{array}{c}\text { Pare- } \\
\text { cido }\end{array} \\
\text { oc. }\end{array}$} & \multirow{2}{*}{$\begin{array}{c}\begin{array}{c}\text { Hipo- } \\
\text { nimia }\end{array} \\
\text { idi. }\end{array}$} & \\
\hline 68 & $-/{ }^{\prime} \mathrm{UN} /-$ & & & & & & & & & 2 \\
\hline 69 & $\digamma^{\prime} \mathrm{U} \theta /-$ & $\mathrm{xx}$ & oc. & idi. & 1 & idi. & & & oc. & 1 \\
\hline 70 & $-\rho^{\prime} \mathrm{U} \theta \mathrm{K} /-$ & $\mathrm{x}$ & idi. & idi. & idi. & oc. & idi. & idi. & 1 & 1 \\
\hline 71 & $-/^{\prime} \mathrm{V} \beta \mathrm{LE} /$ & $\mathrm{xxxxx}^{*}$ & idi. & 1 & idi. & & idi. & & idi. & 1 \\
\hline 72 & -/'VNT/- & $\mathrm{x}$ & 1 & & idi. & idi. & idi. & & & 1 \\
\hline 73 & -/'VNTE/ & $\mathrm{xxxxx}^{*}$ & 1 & idi. & idi. & idi. & idi. & idi. & idi. & 1 \\
\hline 74 & $-/ / \mathrm{V}\} \mathrm{KA} /$ & $x x$ & idi. & (2) & oc. & & & oc. & (1) & 2 \\
\hline 75 & $-/ \mathrm{AK} /-$ & $\mathrm{x}$ & idi. & & oc. & oc. & (1) & idi. & & 1 \\
\hline 76 & $-/ E /-$ & $x x x$ & idi. & & (2) & idi. & (3) & (1) & idi. & 3 \\
\hline 77 & -/FUG/- & $\mathrm{x}$ & 1 & & & & & & & 1 \\
\hline 78 & $-/ J /-$ & $\mathrm{xxxx}^{*}$ & idi. & idi. & idi. & 1 & (2) & idi. & idi. & 2 \\
\hline 79 & -/I'AIDA/ & $\mathrm{x}$ & 1 & & & & & & & 1 \\
\hline 80 & $-/ I K /-$ & $\mathrm{xxxxx}^{*}$ & idi. & idi. & oc. & idi. & 1 & idi. & idi. & 1 \\
\hline 81 & $-/ / \mathrm{LL} /$ & $\mathrm{xx}$ & 1 & idi. & oc. & idi. & oc. & & oc. & 1 \\
\hline 82 & $-/ \mathrm{IM} / /_{1}^{-}$ & $x x$ & & & idi. & & 1 & & & 1 \\
\hline 83 & $-/ \mathrm{IM} / 2^{-}$ & $\mathrm{x}$ & & & & & & & 1 & 1 \\
\hline 84 & $-/ V^{\prime} \beta \bar{U} N D /-$ & $\mathrm{x}$ & 1 & & (2) & & & & & 2 \\
\hline 85 & -/V'DEr/- & $x x x$ & 1 & (2) & & & idi. & & & 2 \\
\hline 86 & -/V'DI $\theta /-$ & $x x x$ & 1 & (2) & idi. & & & & & 2 \\
\hline \multirow[t]{2}{*}{87} & $-/ \mathrm{VN}^{\prime} \mathrm{DEr} /-$ & $\mathrm{x}$ & 1 & idi. & & & & & & 1 \\
\hline & Total & & 28 & 7 & 19 & 23 & 25 & 7 & 16 & 125 \\
\hline
\end{tabular}

3. VALORES ESPECíficos y POLISEMIA SUfiJal

Una vez establecida la lista de nuestros sufijos y de sus valores genéricos, procedimos al estudio de los valores específicos vehiculados por cada uno de ellos, con el fin de determinar los que correspondían a cada sufijo y de ver cuáles de ellos eran polisémicos. Consideramos que un sufijo es polisémico cuando los sufijados a los que da lugar vehiculan diversos valores específicos. Para decidir qué valores específicos debían ser retenidos en el caso de cada sufijo, nos basamos en los mismos criterios estadísticos que utilizamos para determinar los valores genéricos en el caso de cada forma sufijal. Así, retuvimos como valor específico de un sufijo todo valor que caracterizaba $21 \%$ o más de los sufijados que lo contenían, siempre y cuando este valor fuera vehiculado por un mínimo de 3 sufijados derivados. Entre los valores retenidos, distinguimos los valores principales ( $51 \%$ o más de los sufijados) de los valores concurrentes (más de $20 \%$ y menos de $51 \%$ de los sufija- 
dos). Los otros valores fueron considerados, según el caso, como valores específicos ocasionales (más de $10 \%$ y menos de $21 \%$ de los sufijados, correspondiendo siempre a 3 o más sufijados derivados) o idiosincráticos (menos de $11 \%$ de los sufijados o menos de 3 derivados).

Veremos a continuación cómo se distribuye entre los valores específicos que lo caracterizan cada uno de los siete valores genéricos.

\subsection{Los sufijos de agente}

El valor de agente, lo hemos dicho, caracteriza casi una cuarta parte de las lexías adjetivas de nuestro corpus; constituye pues el más importante de los valores vehiculados por los sufijos formadores de adjetivos. Frecuentemente, este valor se aplica a adjetivos que refieren a nociones verbales, pero puede también ser vehiculado por adjetivos que refieren a nociones sustantivas. Se manifiesta como agente efectivo o como agente virtual; el primero, a su vez, puede ser no marcado, causal, habitual o especial; el segundo, puede ser no marcado o causal.

3.1.1. Agente efectivo "no marcado". Este valor caracteriza 1707 de nuestros sufijados, o sea $16 \%$ de los 10524 que constituyen nuestro corpas y es vehiculado por diversas formas sufijales.

Los sufijos no polisémicos que vehiculan este valor como valor principal son: -/'an/2-: charlat-án, holgaz-án, truf-án, etc.; -/' $\mathrm{ete}_{1} / 14$ : agarr-ete, met-ete, chifl-ete, etc.; -/'ika/: llor-ica, quej-ica y acus-ica; - / ' $\mathrm{in}_{2} / 2_{2}-$ : andar-in, bailar-in, hablant-in, etc.; - /'jond ${ }_{2} /-$ : hed-iondo; -/' on ${ }_{1} /-:$ madrug-ón, pregunt-ón, rezong-ón, etc.; -/'or/1-: habl-ador, pele-ador, deline-ador, etc.; -/'or $/ 2^{-:}$protec-tor/triz, director/triz, institu-tor/triz, etc.; -/'Vnt/-: ayud-ant-e/a, estudi-ante-e/a, negoci-ant-e/a, etc.; -/'Vnte/: err-ante, medit-ante, ard-iente, etc. y -/Vn'ßund $1 /$-: err-abundo, medit-abundo, vag-abundo, etcétera.

Los sufijos polisémicos que vehiculan el valor de agente efectivo " no marcado"' como valor principal son: -/' $\mathrm{id}_{2} /-$ : ard-ido, hu-ido, etc.; -/' or $_{1} /$-: comprob-atorio, capt-atorio, destruc-torio, etc. y

${ }^{14}$ La cifra situada a la izquierda de la segunda "/" no aparece más que en el caso de sufijos homonímicos y constituye el coeficiente de hominimia del sufijo. Así, la representación $-/{ }^{\prime} \operatorname{arj}_{1} /$ - será utilizada para el sufijo de "relación", mientras que -/'arj $j_{2} /$ - representará el sufijo de "posesor". 
-/Vn'der/-: cuid-andero, pit-andero, rez-andero, etcétera.

Los sufijos que vehiculan este valor como primer valor concurrente, son los sufijos polisémicos: -/' $\mathrm{er}_{1} /-$ : madrugu-ero, chacotero, cizañ-ero, etc.; -/'i $\beta /$-: ampli-ativo, aprob-ativo, infam-ativo, etc. y $-/ \mathrm{V}^{\prime} \operatorname{der}_{1} /-$ : complac-edero, purific-adero, sal-idero, etcétera.

En fin, el valor de agente efectivo "no marcado" constituye el segundo valor concurrente de los sufijos polisémicos: -/'end/-: lerendo, berr-endo $o_{2}$, distingü-endo; -/' ist $_{1} /-:$ aurr-isto, bañ-isto, tem-isto, etc.; -/' $\mathrm{os}_{2} /$-: apag-oso, dormil-oso, lagrim-oso, etc.; -/il/: péns-il, errátil, puls-átil, etc. $\mathrm{y}-/ \mathrm{V}^{\prime} \operatorname{di} \theta_{1} /-:$ sal-edizo, apart-adizo, vol-adizo, etcétera ${ }^{15}$.

3.1.2. Agente efectivo causal. Nuestro corpus presenta 368 adjetivos sufijados que responden a esta definición, o sea $3.5 \%$ del total de los sufijados.

Los sufijos no polisémicos que vehiculan la noción de agente efectivo causal como valor principal son: $-/^{\prime}$ ifer $_{2} /-$ : frut-ifero, gomifero, luc-ifero, etc.; -/'ifik 1 /-: calor-ifico, mel-ifico, morb-ifico, etc. y -/'oxen/-: lacrim-ógeno/lagrim-ógeno, vaccin-ógeno, pri-ógeno, etcétera.

Son polisémicos y vehiculan este valor como valor principal los sufijos: -l'ist $1 /-$ : barull-isto, brom-isto, pleit-isto, etc. y $-/^{\prime} \mathrm{Os}_{2} /-:$ apetit-oso, delictu-oso, respetu-oso, etcétera.

Como segundo valor concurrente, vehiculan el valor de agente efectivo causal los sufijos -/er $1 /-$ : bull-ero, datil-ero, mal-ero, etc. e $-/^{\prime} \mathrm{i} \beta /-$ : apet-itivo, delict-ivo, respet-ivo, etcétera ${ }^{16}$.

3.1.3. Agente efectivo habitual. Sólo $1 \%$ de los sufijados del corpas (119) responden al valor de agente efectivo habitual. En cuanto a los sufijos aptos para vehicularlo, este valor constituye el valor principal de -/' ista $2 /$ : deport-ista, paracaid-ista, filatel-ista, etc. y el tercer valor concurrente de $-/ \mathrm{er}_{1} /-$ : arep-ero, casamenit-ero, esmerald-ero, etcétera ${ }^{17}$.

15 Ocasionalmente, el valor de agente efectivo "no marcado" es vehiculado por el sufijo $-/$ ista $_{2} /$ : blof-ista.

${ }^{16}$ Ocasionalmente, el valor de agente efectivo causal es vehiculado por los sufijos $-/$ or $j_{1} /-:$ record-atorio y $-/ / \mathrm{Vnt} /-:$ fars-ant-e/a; idiosincráticamente, por los sufijos: - $/ \mathrm{in}_{2} / 2^{-}$: trompadach-in; -/' on ${ }_{1} /-:$ batall-ón $n_{2} ;-/^{\prime}$ or $/_{1^{-}}:$tens-or; -/'Vnte/: combur-ente; -/ill: eréct-il; - $/ \mathrm{V}^{\prime} \beta$ und $_{1} /-:$ nause-abundo, y $-/ \mathrm{V}^{\prime} \operatorname{der}_{1} /-$ : re-idero.

${ }^{17}$ Idiosincráticamente, el valor de agente efectivo habitual es vehiculado también por los sufijos $-/^{\prime}$ ifik $1 /-:$ pac-ifico; $-/^{\prime}$ ist ${ }_{1} /-:$ barat-isto; $-/^{\prime} \mathrm{os}_{2} /-:$ detall-oso;

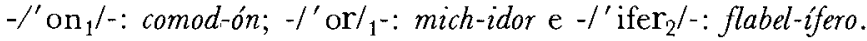


3.1.4. Agente efectivo "especial". Este valor se caracteriza por el hecho de que las diversas paráfrasis que lo constituyen no se aplican sino a sufijados formados por un grupo muy reducido de sufijos: "Que conduce S (mucho)"' (-/'ifer ${ }_{2} /-:$ urin-ifero, aer-ifero, calor-

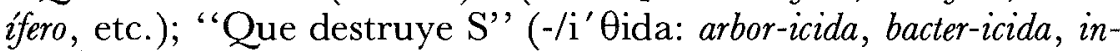
sect-icida, etc.); "Que previene contra $S$ " (-/fug/-: calori-fugo, lucifugo, hidró-fugo, etc.) y "Que V (mucho): perfectivo" (-/' $\mathrm{id}_{2} /-$ : beb-ido, desaparee-ido, padec-ido, etc. y -/' ad/-: chivate-ado, despos-ado, acojon-ado, etc. ${ }^{18}$ ). Sólo 117 sufijados de nuestro corpas, o sea un escaso $1 \%$ de todos los sufijados, responden a este valor.

3.1.5. Agente virtual "no marcado". El agente virtual "no marcado" caracteriza 256 de nuestro sufijados, o sea $2.5 \%$ de los 10524 que constituyen nuestro corpus y es vehiculado como valor principal por los sufijos polisémicos -/il/: nat-átil, contráct-il, móv-il, etc. y $-/ \mathrm{V}^{\prime} \operatorname{di} \theta_{1} /-:$ despeñ-adizo, resbal-adizo, rod-adizo, etc. Como segundo valor concurrente, por $-/ \mathrm{Vn}^{\prime} \mathrm{der} /-$ : vol-andero, colg-andero y parendera; - $/ \mathrm{V}^{\prime} \mathrm{der}_{1} /-$ : desempeñ-adero, resbalad-ero, rod-adero, etc. y -/' or $_{1} /$-: asimil-atorio, evacu-atorio, trasmut-atorio, etc. y, como tercer valor concurrente, por -/'i $\beta /-$ : asimil-ativo, evacu-ativo, trasmut-ativo, etcétera ${ }^{19}$.

3.1.6. Agente virtual causal. Nuestro corpus no presenta más que 33 adjetivos sufijados que responden a esta definición, o sea $0.3 \%$ del total de los sufijados. Sólo el sufijo -/'end/-: rever-endo, horrendo, pud-endo, etc. vehicula esta noción como valor principal ${ }^{20}$.

\subsection{Los sufijos de paciente}

Este valor caracteriza 9\% de las lexías de nuestro corpus y sólo es vehiculado por adjetivos que refieren a una noción verbal.

${ }^{18}$ En el caso de -/'ifer/-, -/i' $\theta$ ida/ y -/fug/- habrá eventualmente que plantear el problema de saber si se trata de sufijos propiamente dichos o, como algunos autores quisieran, de elementos sufijales.

${ }^{19}$ Idiosincráticamente, el valor de agente virtual "no marcado" es vehiculado

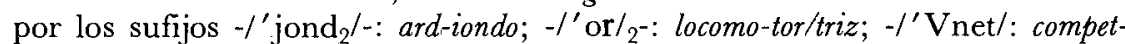

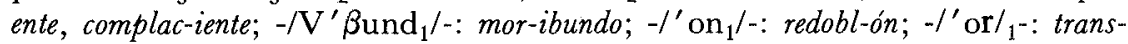
mis-or; e $-/$ ifer $_{2} /-:$ mort-ifero ${ }_{2}$.

${ }^{20}$ Los sufijos $-/{ }^{\prime} \mathrm{id}_{2} /-$ : concurr-ido, -/' $\mathrm{os}_{2} /-$ : rod-oso, $-/$ ifer $_{2} /-:$ mort-ifero ${ }_{2}$, $/ \mathrm{V}^{\prime} \operatorname{der}_{1} /-$ : pos-adero, $-/^{\prime} \operatorname{or}_{j_{1}} /-:$ contempl-atorio, $-/^{\prime} \mathrm{i} \beta /-:$ abus-ivo $o_{2}$ y $-/ \mathrm{V}^{\prime} \mathrm{di} \theta_{1} /-$ : atop-adizo vehiculan el agente virtual causal como valor idiosincrático. 
3.2.1. El paciente efectivo. El valor de paciente efectivo caracteriza 439 sufijados del corpus, o sea un escaso $4 \%$ del conjunto. Es vehiculado como válor principal sólo por los tres sufijos -/' $\mathrm{ad}_{1} /-$ : acandil-ado, afrent-ado, desparram-ado, etc., $-\rho^{\prime} \mathrm{id}_{1} /-:$ aborrec-ido, deseos-ido, imped-ido, etc. y -/'V $\mathrm{ka}_{2} /:$ manus-iashca, guind-ashca, pinz-ashca, etcétera ${ }^{21}$.

3.2.2. El paciente virtual. Este valor caracteriza 470 sufijados (un poco más de 4\%). Sólo los cuatro sufijos -/'and/-: admir-ando, multiplic-ando, vener-ando, etc., -/'Vßle/: mud-able, alter-able, rajable, etc., - $/ \mathrm{V}^{\prime} \mathrm{der}_{2} /-$ : aborrec-edero, cobr-adero, excus-adero, etc. y $-/ \mathrm{V}^{\prime} \mathrm{di} \theta_{2} /$-: ahorr-adizo, alcanz-adizo, compr-adizo, etc., vehiculan este valor como valor principal ${ }^{22}$.

\subsection{Los sufijos de posesor}

El valor de posesor caracteriza $14 \%$ de las lexías de nuestro corpus y sólo es vehiculado por lexías que refieren a una noción sustantiva o numeral.

De las tres paráfrasis que lo representan - "Que tiene $\mathrm{S}(\mathrm{mu}$ cho)", "Que tiene NumS' y "Que tiene S (mucho): padece de" - , sólo la primera es de carácter general y puede ser vehiculada por los diecinueve sufijos siguientes: -/' $\mathrm{ad}_{2} /-$ : aguachin-ado, cochambre-ado, sud-ado, etc.; -/' ar.j $\mathrm{j}_{2} /$-: oliv-ario, celul-ario, millonario, etc.; -/' $\mathrm{a} \theta_{2} /-$ : calzon-azo, cebad-azo, centen-aza, etc.; -/' $\mathrm{e \chi}_{2} /-$ : patul-ejo, grac-ejo, lunar-ejo, etc.; - $/{ }^{\prime} \mathrm{en}_{2} I_{1^{-}}:$mil-eno, doc-eno ${ }_{2}$, veint$e_{2}$, etc.; -/'en $2_{2}^{-}$: cuarent-én, catorc-én, sesent-én, etc.; -/'ent/-: aguach-ento, sudor-iento, corpul-ento; - /'ifer ${ }_{1} /$-: branqu-ifero, palm-

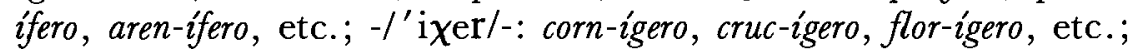
$-/^{\prime}$ jond $_{1} /-$ : cer-iondo, mor-iondo, bot-iondo, etc.; $-/$ 'i $\theta /-$ : cal-izo, pedr-izo, ferr-izo, etc.; -/'on ${ }_{2} /-:$ barrig-ón, colmill-ón, cabez-ón, etc.;

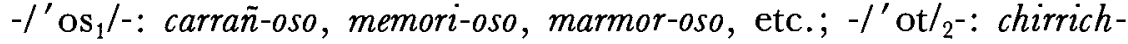
ote, langar-ote, zapal-ote, etc.; -/'uk $/$-: can-uco, pat-uco, timb-uco, etc.; -/'ud/-: dent-udo, juanet-udo, cabell-udo, etc.; -/'uӨ/-: dent-uso /dent-uzo, cacar-uso, cast-uzo, etc.; -/ $\mathrm{e}_{2} /-$ : estertór-eo, férr-eo, marmór-eo, etc. $\mathrm{y}-/ \mathrm{V}^{\prime} \beta \mathrm{und}_{2} /-:$ fur-ibundo, pud-ibundo, sit-ibundo. Este valor

21 Ocasionalmente, el paciente efectivo es vehiculado también por el sufijo -/V' $\mathrm{di}_{2} /$-: vaci-adizo e, idiosincráticamente, por $-/ \mathrm{V}^{\prime} \operatorname{der}_{2} /-$ : serv-idero ${ }_{2}$.

22 - /'V $\int \mathrm{ka}_{2} /$ : manch-ashca, vehicula el valor de paciente virtual idiosincráticamente. 
corresponde a 1305 sufijados de nuestro corpus (12\% del total).

La paráfrasis "Que tiene NumS" no se aplica más que a adjetivos especializados formados con los sufijos $-/^{\prime} \mathrm{en}_{2} /_{1}^{-}$y $-/^{\prime} \mathrm{en} / 2$ a partir de numerales (cincuent-en-o/ $a_{2}$, milen-o/a, catorc-én- $\emptyset / a$, doc-én- $\emptyset / a$, etcétera).

En cuanto al valor de posesor negativo, representado por "Que tiene (mucho): padece de", es más, lo hemos dicho, una modalidad del valor general de posesor, que un valor sufijal propiamente dicho. Sólo ha sido conservado en el caso del sufijo -/'ent/-: almorran-iento, catarr-iento, gran-iento, pioj-iento, etc., que lo vehicula como valor concurrente. En total, este valor puede ser aplicado a 178 sufijados del corpus (1.7\% del total).

\subsection{Los sufijos de identificación}

Este valor genérico caracteriza $18 \%$ de los sufijados del corpus y es vehiculado por 23 sufijos diferentes. No se aplica más que a adjetivos denominales $\mathrm{y}$, principalmente (más de $75 \%$ ), a adjetivos que refieren a nombres propios. Agrupa cinco valores específicos: el origen, la adhesión, la filiación étnica, la descendencia y la localización.

3.4.1. El origen. El valor de origen caracteriza 1481 de nuestros sufijados, sea $14 \%$ de los 10524 que constituyen nuestro corpus y es vehiculado por 21 formas sufijales distintas, como sigue:

Como valor principal, el origen es vehiculado por los sufijos no polisémicos -/' $\mathrm{ak}_{1} /-$ : bosni-aco, egipci-aco, siri-aco, etc.; -/' ajk $\mathrm{k}_{2} /$-: cald-aico, ciren-aico, alt-aico, etc.; -/'an/1-: californi-ano, mexic-ano, silesi-ano, etc.; -/' $\mathrm{e}_{1} /-$ : cald-eo, ciren-eo, europ-eo, etc.; -/'ek/-: tlaxcalt-eco, colim-eco, guatemalt-eco, etc.; -/'eka/: chalm-eca, tuxpan-eca, tlaxcalt-eca, etc.; -/' $\mathrm{eg}_{2} /-$ : leban-iego, aldean-iego, manch-ego, etc.; -/'ense/: canadi-ense, acapulqu-ense, rioplat-ense, etc.; -/' e n/-: sampedr-eño, villarriq-eño, topagu-eño, etc.; -l'es/-: franc-és, nepal-és, santiagu-és, etc.; -/'i/: granad-i, iran-i, nepal-i, etc.; $-/{ }^{\prime} \mathrm{ikola}_{1} /$ : and-ícola, aren-icola, mont-icola, etc.; $-/{ }^{\prime} \mathrm{in}_{1} /{ }_{\mathrm{I}}-$ : abaj-ino, and-ino, santiagu-ino, etc.; $-/^{\prime} \mathrm{m}_{1} / 2^{-}$: florent-in, menorqu-in, placent-in, etc.; -l'isk/-: berber-isco, genov-isco, llan-isco, etc.; -/' ist $_{2} /-$ : belen-isto, llan-isto, vall-isto, etc.; -/'ota/: biarr-ota, cair-ota, chipri-ota, etc.; -/' $\mathrm{un}_{1} /-$ : prad-uno, villaric-uno, lerid-uno, etc. e $-/ \mathrm{j}_{1} /-$ : cipr-io, egip-cio, efes-io, etcétera.

Los sufijos -/' $\mathrm{er}_{2} /-$ : cartagen-ero, jaen-ero, santiagu-ero, etc. e 
-/'ita/: vietnam-ita, ascalon-ita, moscov-ita, etc., polisémicos, lo vehiculan como primer valor concurrente ${ }^{23}$.

3.4.2. La adhesión. Nuestro corpas presenta 221 adjetivos sufijados que responden a este valor, o sea $2 \%$ del total de los sufijadus. Sólo el sufijo $-/$ ' ista $1 /$ : apolinar-ista, bonapart-ista, clasic-ista, etc., no polisémico, vehicula este valor como valor principal; -/' $\mathrm{er}_{2} /$-: chocolat-ero, fald-ero, iglesi-ero, etc. e -/'ita/: adam-ita, jacob-ita, maron-ita, etc., polisémicos, lo vehiculan como segundo valor concurrente ${ }^{24}$.

3.4.3. La filiación étnica. Nuestro corpus presenta 145 adjetivos sufijados que responde a este valor, o sea $1.3 \%$ del total de los sufijados. Sin embargo, ningún sufijo lo vehicula ni como valor principal, ni como valor concurrente ${ }^{25}$.

3.4.4. La descendencia. Sólo 63 sufijados del corpus responden a la definición correspondiente al valor de descendencia. Este valor no constituye el valor principal de ningún sufijo. Sólo -/'ita/: abad-ita, cam-ita, nazar-ita, etc., polisémico, lo vehicula como tercer valor concurrente ${ }^{26}$.

3.4.5. La localización. El valor de localización no caracteriza más que 40 sufijados del corpus. Sólo el sufijo -/'ane/-: limit-áneo, present-áneo, moment-áneo, etc., no polisémico, vehicula este valor como valor principal ${ }^{27}$.

${ }^{23}$ Ocasionalmente, el valor de origen es también vehiculado por el sufijo $-/$ ane/-:" coterr-áneo; idiosincráticamente, por $-/$ 'ista $1 /$ : alban-ista.

${ }^{24}$ Los sufijos $-/^{\prime} \mathrm{ak}_{1} /-:$ isi-aco, $-^{\prime}$ an ${ }^{-}$: hegeli-ano, $-/^{\prime} \mathrm{e}_{1} /-$ : maniqu-eo, -l'ense/: most-ense, -l'e $\mathrm{N} /-:$ cuaresm-eño, $-/$ es/-: agramont-és, $-/{ }^{\prime} \mathrm{i} /:$ fatim-í, $-/^{\prime}$ in $_{1} / 1^{-}$: escuel-ino, $-/$'ist $/ 2$-: concepcio-nisto, $-/^{\prime}$ otal: patri-ota y $/ \mathrm{j}_{1} /-$ : basil-io vehiculan el valor de adhesión idiosincráticamente.

${ }^{25}$ Los sufijos $/^{\prime}$ an $/ 1^{-}:$marroqui-ano y $/^{\prime}$ ek $/-$ : chichim-eco vehiculan el valor de filiación étnica ocasionalmente; -/' eka/: azt-eca; $-/^{\prime} \mathrm{in}_{1} / 2^{-} ;$tocont-ín; $-/^{\prime} \mathrm{er} / 2^{-}$: mesnad-ero; -/'ista $1 /:$ pasion-ista; -/' $\mathrm{e}_{1} /-:$ chip-eo; -/' e $\mathrm{f} /-:$ cabil-eño; -/'es/-: iro$q u$-és; - /'in ${ }_{1} / 1^{-}:$adang-ino; - /'isk/-: gregu-isco e - $/ \mathrm{j}_{1} /-:$ esen-io, lo vehiculan idiosincráticamente.

$26-/^{\prime} \mathrm{ak}_{1} /-:$ mat-aco, taman-aco y arahu-aco, $-/^{\prime} \mathrm{ajk}_{2} /-:$ inc-aico e $-/$ 'i/: abad-i: vehiculan la descendencia como valor ocasional; $-/^{\prime} \mathrm{e}_{1} /-:$ aram-eo $o_{1},-/ \mathrm{ek}^{\prime} \mathrm{e}-:$ chinant-eco, $-/^{\prime} \mathrm{eka} /:$ nahualt-eca, $-/^{\prime} \mathrm{in}_{1} /_{1^{-}}:$ranquel-ino, $-/^{\prime} \mathrm{in}_{1} / 2^{-}:$tocant-in, $-/^{\prime} \mathrm{isk} /-$ : mor-isco e $-/ \mathrm{j}_{1} /-:$ meroving-io, como valor idiosincrático.

${ }^{27}$ Los sufijos $-/$ 'an/ $/ 1^{-}$: danubi-ano, -/' e $/ /$: cas-eño, $-/ \mathrm{er}_{2} /-$ : rincon-era e $-/^{\prime}$ in $_{1} / 1^{-}:$plac-ino, vehiculan la localización como valor idiosincrático. 


\subsection{Los súfijos de parecido}

Este valor genérico caracteriza $7.7 \%$ de nuestros sufijados y es vehiculado por siete sufijos diferentes. De estos sufijos, el más productivo es -/'esk/-: cervant-esco, goy-esco, petrarqu-esco, etc., sufijo que se caracteriza además por el hecho de vehicular el valor específico de parecido modal en vez del de parecido físico. Los otros sufijos de parecido vehiculan el valor específico de parecido físico; de ellos, -l'a ael-: abiet-áceo, borragin-áceo, ros-áceo, etc. es muy productivo, $-/ \mathrm{e}_{3} /-$ : gencian-eo, aroid-eo, etc. y $-/ \mathrm{e}_{1} /-$ : amarilíd-eo, cáct$e o$, córn-eo, etc. son bastante productivos y los otros, $-/$ 'ine/-: abietineo, bix-ineo, laur-ineo, etc., -l' $\mathrm{it}_{2} /-$ : pal-ito, zapat-ito, pingu-ito, etc. $\mathrm{y}-/$ 'oide/: cristal-oide, ov-oide, socialist-oide, etc., son poco productivos. El valor de parecido físico corresponde a 705 sufijados de nuestro corpus ( $7 \%$ del total).

\subsection{Los súfijos de relación}

Este valor genérico caracteriza $21 \%$ de los sufijados del corpas y es vehiculado por 25 sufijos diferentes. Constituye pues el segundo valor más importante de todos los valores vehiculados por nuestros sufijos, tanto desde el punto de vista del número de lexías así definidas como en lo que al número de sufijos que pueden vehicularlo se refiere. Se recordará que bajo este valor hemos agrupado la relación general, la relación numeral general, la relación general ordinal y la relación general partitiva.

3.6.1. Relación general. La relación general caracteriza 2097 de nuestros sufijados, o sea $20 \%$ de los 10524 que constituyen nuestro corpas. Este valor es vehiculado, como valor principal, por los siguientes 22 sufijos no polisémicos: $-/{ }^{\prime} \mathrm{ak}_{2} /-$ : hipocondri-aco, olimpi-aco, simoni-aco, etc.; -l' ajk $\mathrm{k}_{1} /-$ : eb-aico, mos-aico ${ }_{1}$, algebr-aico, etc.; -/'al/: dent-al, palaci-al, dictatori-al, etc.; -/'ar/: cervicul-ar, disciplin-ar, saliv-ar, etc.; $-/^{\prime}$ ar $\mathrm{j}_{1} /-$ : ancil-ario, bili-ario, dent-ario, etc.; -/' $\mathrm{e}_{2} /-$ : albuminoid-eo, coxique-eo, hioid-eo, etc.; -/' $\mathrm{eg}_{1} /-$ : riberiego, palac-iego, frail-ego, etc.; -/'eng/-: abad-engo, real-engo, frailengo, etc.; -/' $\mathrm{er}_{3} /-$ : borregu-ero, cabañ-ero, cerr-ero, etc.; -/' $\mathrm{i} \theta \mathrm{j} /-$ : catedral-icio, cardenal-icio, credit-icio, etc.; $-/$ ikola $_{2} /$ : av-icola, citr-icola, frut-icola; -/' ifik $_{2} /-:$ art-ifico, cer-ifico, espec-ifico; -/' il/: raton-il, potr-il, concej-il, etc.; - ${ }^{\prime}$ in/: carmes-in, lengü-in, rom-in; $-/ \mathrm{in}_{2} / /_{1}^{-}$: bov-ino, ov-ino, estudiant-ino, etc.; $-/$ista $_{3} /$ : catalan-ista, 
falang-ista, moral-ista, etc.; -/'or. $\mathrm{j}_{2} /-$ : suces-orio, arbitr-atorio, censorio, etc.; -/' $\mathrm{un}_{2} /-$ : camer-uno, lacay-uno, paj-uno, etc.; -/ak/-: helespontíraco, olimpíaco, simoni-aco, etc.; $-/ \mathrm{e}_{3} /-$ : albuminóid-eo, origineo, corpór-eo, etc.; -/. $\mathrm{j}_{2} /-$ saturn-io, amazon-io, titan-io, etc. e -/ik/-: amazón-ico, baleár-ico, titán-ico, etcétera ${ }^{28}$.

3.6.2. Relación numeral general. La relación numeral general es representada solamente por once sufijados del corpas. Ningún sufijo la vehicula como valor principal ${ }^{29}$.

3.6.3. Relación numeral ordinal. Este valor se aplica a 66 sufijados del corpus. El sufijo -/'en $/_{1}{ }^{-}$: cent-eno, cuarent-eno, dec-eno, etc., vehicula la relación numeral ordinal como valor principal y el sufijo -/im/ ${ }_{1}^{-}$: cent-ésimo, cuadrag-ésimo, déc-imo, como primer valor concurrente $^{30}$.

3.6.4. Relación numeral partitiva. Este valor se aplica a 64 sufijados del corpus. Es vehiculado como valor principal, por el sufijo -/' a $\beta /$-: cuarent-avo, ochent-avo, treint-avo, etc.; -/im $/_{1^{-}}$: nonag-ésimo, veint-ésimo, cent-ésimo, etc., lo vehicula como primer valor concurrente y $-/^{\prime} \mathrm{en}_{1} /_{1^{-}}$: sept-eno ${ }_{2}$, veint-eno ${ }_{2}$, ochent-eno ${ }_{2}$, etc., como segundo valor concurrente.

\subsection{Los sufijos de hiponimia}

El valor de hiponimia caracteriza 523 sufijados del corpus, sea un escaso $5 \%$ del conjunto. Es vehiculado por los dieciséis sufijos siguientes: -l'ank/-: ru-anco, viej-anco y mal-anco ${ }_{1}$; -l'ang/-: pendango, querind-ango, zangu-ango, etc.; -/' a $\theta_{1} /-$ : bon-azo, flacon-azo, grand-azo, etc.; -/' $\mathrm{e} \chi_{1} /-$ : amarill-ejo, moll-ejo, verd-ejo, etc.; -/'enk/-: azul-enco, flaqu-enco, mud-enco, etc.; -/'ete $2 /$ : gringal-ete, dobl-ete, dulc-ete, etc.; -/'i/-: cans-io, hart-io, crud-io, etc.; -/' $\mathrm{in}_{3} /_{2^{-}}$: comod-in, chipil-in, recent-in, etc.; -/' it ${ }_{1} /$-: alzad-ito, bajon-ito, chicolito, etc.; -/' $\mathrm{on}_{3} /$-: atrevid-ón, picar-ón, dulzarr-ón, etc.; - $/{ }^{\prime} \mathrm{ot} / /_{1^{-}}$: blanc-ote, colorad-ote, franc-ote, etc.; -/' $\mathrm{uk}_{1} /-:$ azul-uco, beat-uco, fe-

${ }^{28}$ Ocasionalmente, la relación general es vehiculada por el sufijo $-/ \mathrm{en}_{1} /{ }_{1}$ : abuch-eno; idiosincráticamente, por el sufijo $-/ \mathrm{im} / 1_{1}:$ : marít-imo.

${ }^{29}$ De manera idiosincrática, la relación numeral general es vehiculada por los sufijos $-/^{\prime} \mathrm{al} /$; catorz-al y $-/{ }^{\prime} \operatorname{arj}_{1} /-$ : decen-ario.

${ }^{30}$ Los sufijos -/arj $\mathrm{j}_{1} /-:$ terci-ario, $-/ \mathrm{\prime}^{\mathrm{er}} \mathrm{r}_{3} /$ : tere-ero e $-/ \mathrm{j}_{2} /-:$ terc-io, vehiculan la relación numeral ordinal idiosincráticamente. 


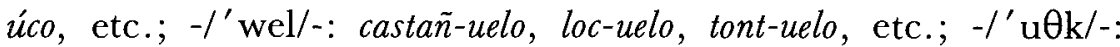
negr-uzco, leper-usco ${ }_{1}$, pard-usco, etc.; -/'V $\left[\mathrm{ka}_{1} /:\right.$ mojos-iashca, leloyashca, changu-yashca, etc. e -/im/ $/ 2^{-}$: lev-ísimo, finit-imo, universalisimo, etcétera.

Se notará que once de estos sufijos $\left(-/^{\prime} \mathrm{a} \theta_{1} /-,-/^{\prime} \mathrm{e} \chi_{1} /-\right.$,

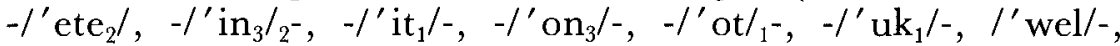
-/'u $\mathrm{u} \mathrm{k} / \mathrm{-}$ e -/im $\left./ 2^{-}\right)$son homónimos de sufijos normalmente aceptados como sufijos diminutivos, aumentativos, superlativos o despectivos en.español (Gooch 1970).

Hemos de concluir pues que en nuestro corpus la hiponimia es casi un valor marginal. Los 430 adjetivos del corpus que responden a esta paráfrasis fueron conservados porque las fuentes lexicográficas no indican francamente el valor ponderativo del adjetivo. Éste se debe, en la mayor parte de los casos, a que este valor ponderativo está impregnado de una cierto grado de lexicalización, contrariamente a lo que sucede cuando se trata del uso común de los sufijos apreciativos (Alonso 1961, Gooch 1970 y Faitelson-Weiser 1980, pp. 184-247). Así, si se compara un adjetivo como blanquito, derivado de blanco, con un adjetivo como blanquizo, derivado del mismo adjetivo, se constata que la diferencia esencial entre los dos consiste en la libertad de su uso. En efecto, mientras que el primero (normalmente ausente de los diccionarios del español) puede ser usado en casi cualquier circunstancia para expresar un grado más o menos intenso de blanco -tanto al nivel denotativo como connotativo-, la utilización del segundo, definido como "que tira a blanco" se ve limitada a contextos precisos en los que denotativamente, se quiere expresar una aproximación de blanco. Lo mismos sucede con viejanco comparado con viejito, azulenco con azulillo, etcétera.

Lo que acabamos de decir, se resume en el cuadro 5.

Cuadro 5

Los sufijos formadores de adjetivos $-y$ sus valores

\begin{tabular}{|c|c|c|c|c|c|c|}
\hline & \multicolumn{2}{|c|}{ Sufijo } & \multirow[b]{2}{*}{$\begin{array}{c}\text { Valores } \\
\text { genéricos }\end{array}$} & \multirow[b]{2}{*}{$\begin{array}{c}\text { Polisemia } \\
+/-\end{array}$} & \multirow[b]{2}{*}{$\begin{array}{l}\text { Valores } \\
\text { especificos }\end{array}$} & \multirow[b]{2}{*}{$\begin{array}{l}\text { Rango del } \\
\text { valor }\end{array}$} \\
\hline & $\begin{array}{l}\text { Represen- } \\
\text { tación }\end{array}$ & $\begin{array}{l}\text { Producti- } \\
\text { vidad }\end{array}$ & & & & \\
\hline 1 & $-/ \mathrm{ak}_{1} /-$ & $\mathrm{xxx}$ & Id & - & orig. & ppal. \\
\hline 2 & $-/ ' \mathrm{ak}_{2} /-$ & $x x$ & Rel & - & gen. & ppal. \\
\hline 3 & $-{ }^{\prime} \mathrm{a} \theta \mathrm{e} /-$ & $\mathbf{x x x x x}$ & Par & - & físico & ppal. \\
\hline 4 & $-/ ' \operatorname{ad}_{1} /-$ & $\mathrm{xxxxx^{* }}$ & Pac & - & efect. & ppal. \\
\hline 5 & $-/{ }^{\prime} \operatorname{ad}_{2} /-$ & $\mathrm{xxxx} *$ & Pos & - & efect. $\emptyset$ & ppal. \\
\hline
\end{tabular}


Cuadro 5 (continúa)

\begin{tabular}{|c|c|c|c|c|c|c|}
\hline & & & & & & \\
\hline & $\begin{array}{l}\text { Represen- } \\
\text { tación }\end{array}$ & $\begin{array}{l}\text { Producti- } \\
\text { vidad }^{31}\end{array}$ & $\begin{array}{c}\text { Valores } \\
\text { genéricos }\end{array}$ & $\begin{array}{l}\text { Polisemia } \\
+/-\end{array}$ & $\begin{array}{l}\text { Valores } \\
\text { especificos } 33\end{array}$ & $\begin{array}{l}\text { Rango del } \\
\text { valor } 34\end{array}$ \\
\hline 6 & $-/^{\prime} \mathrm{ajk}_{1} /-$ & $\mathrm{xx}$ & Rel & - & gen. & ppal. \\
\hline 7 & $-/{ }^{\prime}$ ajk $k_{2} /-$ & $\mathrm{x}$ & Id & - & orig. & ppal. \\
\hline 8 & $-/{ }^{\prime} \mathrm{al} /{ }^{\prime}$ & $\mathrm{xxxxx}^{*}$ & Rel & - & gen. & ppal. \\
\hline 9 & $-f^{\prime}$ àn/ $1^{-}$ & $\operatorname{xxxxx}^{*}$ & Id & - & orig. & ppal. \\
\hline 10 & $-/^{\prime}$ an $/ 2^{-}$ & $\mathrm{xx}$ & $\mathrm{Ag}$ & - & efect. $\emptyset$ & CONC. \\
\hline 11 & - /'ank/- & $\mathrm{x}$ & $\mathrm{Hi}$ & - & & CONC. \\
\hline 12 & $-/$ ' and/- & $\mathrm{x}$ & Pac & - & virt. & ppal. \\
\hline 13 & $-l^{\prime}$ ane/- & $x x$ & Id & - & localiz. & ppal. \\
\hline 14 & -/'ang/- & $\mathrm{x}$ & $\mathrm{Hi}$ & - & & ppal. \\
\hline 15 & $-/^{\prime} \mathrm{ar} /$ & $\mathrm{xxxxx}$ & Rel & - & gen. & ppal. \\
\hline 16 & $-l^{\prime} \operatorname{ar}_{j_{1}} /-$ & $\operatorname{xxxxx}^{*}$ & Rel & - & gen & ppal. \\
\hline 17 & $-/^{\prime} \operatorname{ar} j_{2} /-$ & $\mathrm{xxxx}^{*}$ & Pos & - & efect. $\varnothing$ & ppal. \\
\hline 18 & $-/^{\prime} \mathrm{a} \beta /-$ & $\mathrm{xxx}$ & Rel & - & num. partit. & ppal. \\
\hline 19 & $-{ }^{\prime} \mathrm{a} \theta_{1} /-$ & $\mathrm{xx}$ & $\mathrm{Hi}$ & - & & ppal. \\
\hline 20 & $-{ }^{\prime} \mathrm{a} \theta_{2} /-$ & $\mathrm{x}$ & Pos & - & efect. $\varnothing$ & ppal. \\
\hline 21 & $-J^{\prime} \mathrm{e}_{1} /-$ & $x x x$ & Id & - & orig. & ppal. \\
\hline 22 & $-f^{\prime} e_{2} /-$ & $\mathrm{xxx}$ & Rel & - & gen. & ppal. \\
\hline 23 & $-l^{\prime} \mathrm{e}_{3} /-$ & $\mathrm{xxx}$ & Par & - & fúsico & ppal. \\
\hline 24 & -/'ek/- & $\mathrm{xxxx}$ & Id & - & orig. & ppal. \\
\hline 25 & -l'ekal & $\mathrm{xx}$ & Id & - & orig. & ppal. \\
\hline 26 & $-l^{\prime} \mathrm{eg}_{1} /-$ & $x x x$ & Rel & - & gen & ppal. \\
\hline 27 & $-{ }^{\prime} \mathrm{eg}_{2} /-$ & $\mathrm{xx}$ & Id & - & orig. & ppal. \\
\hline 28 & $-l^{\prime} \mathrm{ex}_{1} /-$ & $x x$ & $\mathrm{Hi}$ & - & & ppal. \\
\hline 29 & $-\gamma^{\prime} e \chi_{2} /-$ & $\mathrm{x}$ & Pos & - & efect. $\emptyset$ & ppal. \\
\hline 30 & $-/^{\prime} \mathrm{en}_{1} / 1^{-}$ & $\operatorname{xxxx}$ & Rel & + & $\begin{array}{l}\text { num. ord. } \\
\text { num. partit. }\end{array}$ & $\begin{array}{l}\text { ppal. } \\
\text { cone. } 1\end{array}$ \\
\hline 31 & $-/^{\prime} \mathrm{en}_{2} /{ }^{-}$ & $\mathrm{xx}$ & Pos & - & efect. $\varnothing$ & ppal. \\
\hline 32 & $-l^{\prime} \mathrm{en} / 2^{-}$ & $\mathrm{x}$ & Pos & - & efect. $\varnothing$ & ppal. \\
\hline 33 & -/'enk/- & $\mathrm{xx}$ & $\mathrm{Hi}$ & - & & ppal. \\
\hline 34 & $-/$ end/- & $\mathrm{x}$ & $\mathrm{Ag}$ & + & $\begin{array}{l}\text { virt. causal } \\
\text { efect. } \emptyset\end{array}$ & $\begin{array}{l}\text { CONC. } 1 \\
\text { CONC. } 2\end{array}$ \\
\hline
\end{tabular}

${ }^{31} \mathrm{xxxxx}=100$ sufijados o más en el corpus; $\mathrm{xxxx}=$ entre 50 y 99 sufijados en el corpus, $\mathrm{xxx}=$ entre 25 y 49 sufijados en el corpus; $\mathrm{xx}=$ entre 10 y 24 sufijados en el corpus, $\mathrm{x}=$ menos de 10 sufijados en el corpus. El ${ }^{*}$ que sigue una secuencia de " $x$ " indica que el sufijo en cuestión ha sido estudiado a partir de un muestreo.

${ }^{32} \mathrm{Ag}=$ agente; $\mathrm{Hi}=$ hiponimia $; \mathrm{Id}=$ identificación; $\mathrm{Pac}=$ paciente $; \mathrm{Par}$ = parecido; Pos = posesor $;$ Rel = relación.

${ }^{33}$ Adh. = adhesión; descend. = descendencia; efect. = efectivo; "esp." = especial; gen. = general; habit. = habitual; localiz. = localización; neg. = negativo; num. $=$ numeral $;$ ord.$=$ ordinal $;$ orig. $=$ origen $;$ partit. $=$ partitivo; virt. $=$ virtual; $\phi=$ no marcado .

${ }^{34}$ Conc. $=$ concurrente; ppal. $=$ principal. 
Cuadro 5 (continúa)

\begin{tabular}{|c|c|c|c|c|c|c|}
\hline & \multicolumn{2}{|c|}{ Sufijo } & \multirow[b]{2}{*}{$\begin{array}{l}\text { Valores } \\
\text { genéricos }\end{array}$} & \multirow[b]{2}{*}{$\begin{array}{c}\text { Polisemia } \\
+/-\end{array}$} & \multirow[b]{2}{*}{$\begin{array}{l}\text { Valores } \\
\text { especificos }\end{array}$} & \multirow[b]{2}{*}{$\begin{array}{l}\text { Rango del } \\
\text { valor }\end{array}$} \\
\hline & $\begin{array}{l}\text { Represen- } \\
\text { tación }\end{array}$ & $\begin{array}{l}\text { Producti- } \\
\text { vidad }\end{array}$ & & & & \\
\hline 35 & -/'eng/- & $\mathrm{x}$ & Rel & - & gen. & CONC. \\
\hline 36 & $-/$ 'ense/ & $\mathrm{xxxxx}^{*}$ & Id & - & orig. & ppal. \\
\hline 37 & $-I^{\prime}$ ent $/-$ & $\mathbf{x x x x}$ & Pos & + & $\begin{array}{l}\text { efect. } \emptyset \\
\text { efect. neg. }\end{array}$ & $\begin{array}{l}\text { ppal. } \\
\text { conc. } 2\end{array}$ \\
\hline 38 & -/'en/- & $\mathrm{xxxxx}^{*}$ & Id & - & orig. & ppal. \\
\hline 39 & $-/ \mathrm{er}_{1} /-$ & $\mathrm{xxxxx}^{*}$ & $\mathrm{Ag}$ & + & $\begin{array}{l}\text { efect. } \emptyset \\
\text { efect. causal } \\
\text { efect. habit. }\end{array}$ & $\begin{array}{l}\text { conc. } 1 \\
\text { conc. } 2 \\
\text { conc. } 3\end{array}$ \\
\hline 40 & $-I^{\prime} \mathrm{er}_{2} /-$ & $\mathrm{xxxx}^{*}$ & Id & + & $\begin{array}{l}\text { orig. } \\
\text { adh. }\end{array}$ & $\begin{array}{l}\text { conc. } 1 \\
\text { conc. } 2\end{array}$ \\
\hline 41 & $-\mathrm{H}^{\prime} \mathrm{er}_{3} /$ & $\mathrm{xxxx}^{*}$ & Rel & - & gen. & ppal. \\
\hline 42 & $-/$ es/- & $\operatorname{xxxxx}$ & Id & - & orig. & ppal. \\
\hline 43 & 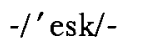 & $\operatorname{xxxxx}$ & Par & - & modal & ppal. \\
\hline 44 & $-/^{\prime}$ ete $_{1} /$ & $\mathrm{xx}$ & $\mathrm{Ag}$ & - & efect. $\varnothing$ & ppal. \\
\hline 45 & $-I^{\prime}$ ete $_{2} /$ & $x \mathbf{x}$ & $\mathrm{Hi}$ & - & & ppal. \\
\hline 46 & $-/^{\prime} \mathrm{i} /$ & $x x x x$ & Id & - & orig. & ppal. \\
\hline 47 & $-/^{\prime} \mathrm{i} /-$ & $\mathrm{xx}$ & $\mathrm{Hi}$ & - & & CONC. \\
\hline 48 & $-/$ ika/ & $\mathrm{x}$ & $\mathrm{Ag}$ & - & efect. $\emptyset$ & CONC. \\
\hline 49 & $-\mu^{\prime} \mathrm{i} \phi \mathrm{j} /-$ & $\mathrm{xxx}$ & Rel & - & gen. & ppal. \\
\hline 50 & $-/$ 'ikola $/$ & $\mathrm{xx}$ & Id & - & orig. & ppal. \\
\hline 51 & -/'ikola ${ }_{2} /$ & $\mathrm{xx}$ & Rel & - & gen. & ppal. \\
\hline 52 & $-/ \mathrm{id}_{1} /-$ & $\mathrm{xxxxx}^{*}$ & Pac & - & efect. & ppal. \\
\hline 53 & $-/ \mathrm{id}_{2} /-$ & $\operatorname{xxxxx}^{*}$ & $\mathrm{Ag}$ & + & $\begin{array}{l}\text { efec. } \emptyset \\
\text { efect. "esp.", }\end{array}$ & $\begin{array}{l}\text { ppal. } \\
\text { conc. } 2\end{array}$ \\
\hline 54 & $-/$ ifer $_{1} /-$ & $\operatorname{xxxx}$ & Pos & - & efect. $\emptyset$ & ppal. \\
\hline 55 & $-l^{\prime}$ ifer $_{2} /-$ & $\mathrm{xxx}$ & $\mathrm{Ag}$ & - & efect. causal & ppal. \\
\hline 56 & $-/$ ifik $_{1} /-$ & $x x$ & $\mathrm{Ag}$ & - & efect. causal & ppal. \\
\hline 57 & $-/{ }^{\prime}$ ifik $k_{g} /-$ & $\mathrm{x}$ & Rel & - & gen & ppal. \\
\hline 58 & $-/$ ixer/- & $\mathrm{x}$ & Pos & - & efect. $\emptyset$ & ppal. \\
\hline 59 & $-/^{\prime} \mathrm{il} /$ & $\mathrm{xxxx}$ & Rel & - & gen. & ppal. \\
\hline 60 & $-/$ in/ & $\mathrm{x}$ & Rel & - & gen. & CONC. \\
\hline 61 & $-/ \operatorname{in}_{1} / 1^{-}$ & $\mathrm{xxxx}^{*}$ & Id & - & orig. & ppal. \\
\hline 62 & $-/^{\prime} \operatorname{in}_{2} / 1^{-}$ & $\mathrm{xxx}^{*}$ & Rel & - & gen. & ppal. \\
\hline 63 & $-/^{\prime} \operatorname{in}_{1} / 2_{2}^{-}$ & $\mathrm{xx}$ & Id & - & orig. & ppal. \\
\hline 64 & $-/^{\prime} \operatorname{in}_{2} / 2^{-}$ & $\mathrm{xx}$ & $\mathrm{Ag}$ & - & efect. $\emptyset$ & ppal. \\
\hline 65 & $-/^{\prime}$ in $_{3} / 2^{-}$ & $\mathrm{xx}$ & $\mathrm{Hi}$ & - & & ppal. \\
\hline 66 & $-/$ inel- & $\mathrm{xx}$ & Par & - & físico & ppal. \\
\hline 67 & $-/$ jond $_{1} /-$ & $\mathrm{x}$ & Pos & - & efect. $\emptyset$ & ppal. \\
\hline 68 & $-/^{\prime}$ jond $\mathrm{d}_{2} /-$ & $\mathrm{x}$ & $\mathrm{Ag}$ & - & efect. $\emptyset$ & Ppal. \\
\hline 69 & $-J^{\prime}$ isk $/-$ & $x x$ & Id & - & orig. & ppal. \\
\hline 70 & $-/$ ist $_{1} /-$ & $\mathrm{xxx}$ & $\mathrm{Ag}$ & + & $\begin{array}{l}\text { efect. causal } \\
\text { efect. } \emptyset\end{array}$ & $\begin{array}{l}\text { ppal. } \\
\text { conc. } 2\end{array}$ \\
\hline 71 & $-/$ ist $_{2} /-$ & $\mathrm{xx}$ & Id & - & orig. & ppal. \\
\hline 72 & $-/$ ista $_{1} /$ & $\mathrm{xxxx}^{*}$ & Id & - & adh. & ppal. \\
\hline
\end{tabular}


Cuadro 5 (continúa)

\begin{tabular}{|c|c|c|c|c|c|c|}
\hline & & & & & & \\
\hline & $\begin{array}{l}\text { Represen- } \\
\text { tación }\end{array}$ & $\begin{array}{l}\text { Producti- } \\
\text { vidad }\end{array}$ & $\begin{array}{c}\text { Valores } \\
\text { genéricos }\end{array}$ & $\begin{array}{c}\text { Polisemia } \\
+/-\end{array}$ & $\begin{array}{l}\text { Valores } \\
\text { especificos }\end{array}$ & $\begin{array}{l}\text { Rango del } \\
\text { valor }\end{array}$ \\
\hline 73 & $-/$ 'ista ${ }_{2} /$ & $\mathrm{xxxx}^{*}$ & $\mathrm{Ag}$ & - & efect. habit. & ppal. \\
\hline 74 & $-/$ ista ${ }_{3} /$ & $\mathrm{xxx}^{*}$ & Rel & - & gen. & ppal. \\
\hline 75 & $-/$ it $_{1} /-$ & $\mathrm{xxx}$ & $\mathrm{Hi}$ & - & & ppal. \\
\hline 76 & $-/ \mathrm{it}_{2} /-$ & $\mathrm{xx}$ & Par & - & fśico & ppal. \\
\hline 77 & $-/$ ital & $\operatorname{xxxx}$ & Id & + & $\begin{array}{l}\text { orig. } \\
\text { adh. } \\
\text { descend. }\end{array}$ & $\begin{array}{l}\text { conc. } 1 \\
\text { conc. } 2 \\
\text { conc. } 3\end{array}$ \\
\hline 78 & $-\nu^{\prime} \mathrm{i} \beta /-$ & $\mathrm{xxxxx}^{*}$ & Ag & + & $\begin{array}{l}\text { efect. } \emptyset \\
\text { efect. causal } \\
\text { virt. } \emptyset\end{array}$ & $\begin{array}{l}\text { conc. } 1 \\
\text { conc. } 2 \\
\text { conc. } 3\end{array}$ \\
\hline 79 & $-/^{\prime} \mathrm{i} \theta /-$ & $\mathrm{xx}$ & Pos & - & efect. $\emptyset$ & CONC. \\
\hline 80 & -l'oxen/- & $\mathrm{xx}$ & $\mathrm{Ag}$ & - & efect. causal & ppal. \\
\hline 81 & -/'ojde/ & $\mathrm{xx}$ & Par & - & físico & ppal. \\
\hline 82 & $-/$ 'on ${ }_{1} /-$ & $\mathrm{xxxxx}^{*}$ & $\mathrm{Ag}$ & - & efect. $\emptyset$ & ppal. \\
\hline 83 & $-/{ }^{\prime}$ on $_{2} /-$ & $\mathrm{xxxx}^{*}$ & Pos & - & efect. $\emptyset$ & ppal. \\
\hline 84 & $-/{ }^{\prime} \mathrm{on}_{3} /-$ & $\mathrm{xxxx}^{*}$ & $\mathrm{Hi}$ & - & & ppal. \\
\hline 85 & $-/$ or $/ t^{-}$ & $\mathrm{xxxxx}^{*}$ & $\mathrm{Ag}$ & - & efect. $\varnothing$ & ppal. \\
\hline 86 & $-/^{\prime}$ or $/ 2^{-}$ & $\mathrm{x}$ & $\mathrm{Ag}$ & - & efect. $\emptyset$ & ppal. \\
\hline 87 & $-/{ }^{\prime}$ or $j_{1} /-$ & $\operatorname{xxxxx}$ & $\mathrm{Ag}$ & + & $\begin{array}{l}\text { efect. } \emptyset \\
\text { virt. }\end{array}$ & $\begin{array}{l}\text { ppal. } \\
\text { conc. } 2\end{array}$ \\
\hline 88 & $-/{ }^{\prime}$ or $\mathrm{j}_{2} /-$ & $\mathrm{xxxx}$ & Rel & - & gen. & ppal. \\
\hline 89 & $-/$ os $_{1} /-$ & $\mathrm{xxxxx}^{*}$ & Pos & - & efect. $\varnothing$ & ppal. \\
\hline 90 & $-/$ os $/-$ & $\mathrm{xxxx}^{*}$ & $\mathrm{Ag}$ & + & $\begin{array}{l}\text { efect. causal } \\
\text { efect. } \emptyset\end{array}$ & $\begin{array}{l}\text { ppal. } \\
\text { conc. } 2\end{array}$ \\
\hline 91 & $-l^{\prime}$ ot $/ 1^{-}$ & $\mathrm{xx}$ & $\mathrm{Hi}$ & - & & ppal. \\
\hline 92 & $-l^{\prime}$ ot $/ 2^{-}$ & $\mathrm{x}$ & Pos & - & efect. $\varnothing$ & CONC. \\
\hline 93 & $-/$ otal & $x \mathrm{x}$ & Id & & orig. & ppal. \\
\hline 94 & $-/^{\prime} \mathrm{uk}_{1} /-$ & $\mathrm{x}$ & $\mathrm{Hi}$ & - & & ppal. \\
\hline 95 & $-\gamma^{\prime} \mathrm{uk}_{2} /-$ & $\mathrm{x}$ & Pos & - & efect. $\varnothing$ & ppal. \\
\hline 96 & $-/ \mathrm{l}^{\prime} \mathrm{ud} /-$ & $\mathrm{xxxxx}^{*}$ & Pos & - & efect. $\varnothing$ & ppal. \\
\hline 97 & $-f^{\prime \prime}$ wel/- & $\mathrm{x}$ & $\mathrm{Hi}$ & - & & ppal. \\
\hline 98 & $-/ \operatorname{un}_{1} /-$ & $\operatorname{xxxx}$ & Id & - & orig. & ppal. \\
\hline 99 & $-/^{\prime} \mathrm{un}_{2} /-$ & $\mathrm{xxx}$ & Rel & - & gen. & ppal. \\
\hline 100 & $-/^{\prime} \mathrm{u} \theta /-$ & $\mathrm{xx}$ & Pos & - & efect. $\varnothing$ & ppal. \\
\hline 101 & $-/^{\prime} \mathrm{u} \theta \mathrm{k} /-$ & $\mathrm{xx}$ & $\mathrm{Hi}$ & - & & ppal. \\
\hline 102 & $-/^{\prime} \mathrm{V} \beta \mathrm{le} /$ & $\mathrm{xxxxx}^{*}$ & Pac & - & virt. & ppal. \\
\hline 103 & $-l^{\prime} \mathrm{Vnt} /-$ & $\mathrm{xx}$ & $\mathrm{Ag}$ & - & efect. $\emptyset$ & ppal. \\
\hline 104 & $-f^{\prime}$ Vntel & $\mathrm{xxxxx}^{*}$ & Ag & - & efect. $\emptyset$ & ppal. \\
\hline 105 & $-/^{\prime} \mathrm{V} \mid \mathrm{ka}_{1} /$ & $\mathrm{xxx}$ & $\mathrm{Hi}$ & - & & ppal. \\
\hline 106 & $-/^{\prime} \mathrm{V}\left\lceil\mathrm{ka}_{2} /\right.$ & $x x x$ & Pac & - & efect. & ppal. \\
\hline 107 & $-/ \mathrm{ak} /-$ & $\mathrm{xx}$ & Rel & - & gen. & CONC. \\
\hline 108 & $-/ e_{1} /-$ & $\mathrm{xxx}$ & Par & - & físico & ppal. \\
\hline 109 & $-/ e_{2} /-$ & $\mathrm{xxx}$ & Pos & - & efect. $\emptyset$ & ppal. \\
\hline 110 & $-/ e_{3} /-$ & $x x x$ & Rel & - & gen. & ppal. \\
\hline
\end{tabular}


CUADRo 5 (concluye)

\begin{tabular}{|c|c|c|c|c|c|c|}
\hline & \multicolumn{2}{|c|}{ Súfijo } & \multirow[b]{2}{*}{$\begin{array}{c}\text { Valores } \\
\text { genéricos }\end{array}$} & \multirow[b]{2}{*}{$\begin{array}{c}\text { Polisemia } \\
+/-\end{array}$} & \multirow[b]{2}{*}{$\begin{array}{l}\text { Valores } \\
\text { especificicos }\end{array}$} & \multirow[b]{2}{*}{$\begin{array}{l}\text { Rango del } \\
\text { valor }\end{array}$} \\
\hline & $\begin{array}{l}\text { Represen- } \\
\text { tación }\end{array}$ & $\begin{array}{l}\text { Producti- } \\
\text { vidad }\end{array}$ & & & & \\
\hline 111 & -/fug/- & $\mathrm{xx}$ & $\mathrm{Ag}$ & - & efect." esp." & ppal. \\
\hline 112 & $-/ j_{1} /-$ & $x x x x x^{*}$ & Id & - & orig. & ppal. \\
\hline 113 & $-/ \mathrm{j}_{2} /-$ & $\mathrm{xxx}^{*}$ & Rel & - & gen. & ppal. \\
\hline 114 & - /i'Oidal $^{\prime}$ & $\mathrm{xx}$ & $\mathrm{Ag}$ & - & efect."esp." & ppal. \\
\hline 115 & $-/ \mathrm{ik} /-$ & $\operatorname{xxxxx}^{*}$ & Rel & - & gen. & ppal. \\
\hline 116 & $-/ \mathrm{il} /$ & $\mathrm{xx}$ & $\mathrm{Ag}$ & + & $\begin{array}{l}\text { virt. } \emptyset \\
\text { efect. } \emptyset\end{array}$ & $\begin{array}{l}\text { ppal. } \\
\text { conc. } 2\end{array}$ \\
\hline 117 & $-/ \mathrm{im} / 1^{-}$ & $\operatorname{xxxx}$ & Rel & + & $\begin{array}{l}\text { num. ord. } \\
\text { num. partit. }\end{array}$ & $\begin{array}{l}\text { conc. } 1 \\
\text { conc. } 1\end{array}$ \\
\hline 118 & $-/ \mathrm{im} / 2^{-}$ & $\mathrm{xx}$ & $\mathrm{Hi}$ & - & & ppal. \\
\hline 119 & $-/ V^{\prime} \beta$ und $_{1} /-$ & $\mathrm{x}$ & $\mathrm{Ag}$ & - & efect. $\varnothing$ & ppal. \\
\hline 120 & $-/ V^{\prime} \beta$ und $_{2} /-$ & $\mathrm{x}$ & Pos & - & efect. $\varnothing$ & ppal. \\
\hline 121 & $-/ V^{\prime} \operatorname{der}_{1} /-$ & $\mathrm{xxxx}$ & $\mathrm{Ag}$ & + & $\begin{array}{l}\text { efect. } \varnothing \\
\text { virt. } \emptyset\end{array}$ & $\begin{array}{l}\text { conc. } 1 \\
\text { conc. } 2\end{array}$ \\
\hline 122 & $-/ V^{\prime} \operatorname{der}_{2} /-$ & $\mathrm{xxxx}$ & $\mathrm{Pac}$ & - & virt. & ppal. \\
\hline 123 & $-/ V^{\prime} \operatorname{di} \theta_{1} /-$ & $\mathrm{xxxx}$ & $\mathrm{Ag}$ & + & $\begin{array}{l}\text { virt. } \emptyset \\
\text { efect. } \emptyset\end{array}$ & $\begin{array}{l}\text { ppal. } \\
\text { conc. } 2\end{array}$ \\
\hline 124 & $-/ V^{\prime} \operatorname{di} \theta_{2} /-$ & $\operatorname{xxxx}$ & $\mathrm{Pac}$ & - & virt. & ppal. \\
\hline 125 & $-/ V n^{\prime} \operatorname{der} /-$ & $\mathrm{xx}$ & $\mathrm{Ag}$ & + & $\begin{array}{l}\text { efect. } \emptyset \\
\text { virt. } \emptyset\end{array}$ & $\begin{array}{l}\text { ppal. } \\
\text { conc. } 2\end{array}$ \\
\hline
\end{tabular}

Hemos pues postulado:

a. Que el sistema de sufijos formadores de adjetivos en español moderno se presenta como un sistema rico en formas: identificamos 125 sufijos formadores de adjetivos que emanan de 87 formas sufijales diferentes.

$b$. Que cada sufijo se distingue de los demás por su forma y su valor genérico.

c. Que, en su conjunto, estos sufijos vehiculan siete valores genéricos diferentes: el valor de agente, el valor de relación, el valor de identificación, el valor de posesor, el valor de paciente, el valor de parecido y el valor de hiponimia.

d. Que en el caso de la hiponimia, los sufijos no desempeñan plenamente su función sufijal, puesto que no hay traslación de un campo morfosemántico a otro.

e. Que cuando dos o más sufijos emanan de la misma forma sufijal y no se distinguen más que por su valor genérico, se trata de sifijos homonimicos.

$f$. Que 32 de nuestras formas sufijales dan lugar a sufijos homonímicos: agente y relación: -/'IFIK/-y -/'OrJ/-; agente e identifi- 
cación: -/'IST/-; agente y posesor: -/V' $\beta \mathrm{UND} /-$; agente y paciente: -/V'DI $\theta /-$ y -/V'DEr/-; agente e hiponimia: -/'ETE/; relación e identificación: -/'AJK/- y -/'EG/-; relación y posesor: -/'ArJ/- y

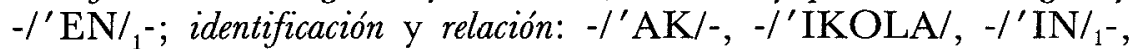
-/'UN/- e -/J/-; posesor y agente: -/'IFEr/-, -/"JOND/- y -/'OS/-; paciente y agente: $-l^{\prime} \mathrm{ID} /-;$ paciente y posesor: -l' $\mathrm{AD} /-;$ hiponimia y posesor: -/" $\mathrm{A} \theta /-,-/^{\prime} \mathrm{UK} /-\mathrm{y}-/^{\prime} \mathrm{E} \chi /-;$ hiponimia y paciente: -/' $\mathrm{V} \int \mathrm{KA} /$; hiponimia y parecido: -/'IT/-; agente, identificación y relación; -/' $\mathrm{Er} /-$; agente, posesor e hiponimia: -/'ON/-; identificación, agente y relación: -/'ISTA/; identificación, relación y parecido: -/'E/-; identificación, agente e hiponimia: -/' IN/ ${ }_{2}^{-}$y, en fin, parecido, posesor y relación: -/E/-.

$g$. Que los siete valores genéricos se dejan analizar en 21 valores específicos: agente efectivo "no marcado", agente efectivo causal, agente efectivo habitual, agente efectivo "especial", agente virtual "no marcado", agente virtual causal, relación general, relación numeral ordinal, relación numeral partitiva, relación numeral general, origen, adhesión, filiación étnica, descendencia, localización, posesor, paciente efectivo, paciente virtual, parecido físico, parecido modal y, en fin, hiponimia.

$h$. Que, normalmente, estos valores específicos son vehiculados por los distintos sufijos como valores principales o como valores concurrentes, pero que un sufijo puede también vehicular ciertos valores ocasional o idiosincráticamente.

$i$. Que cuando un sufijo vehicula, como valor principal o como valor concurrente, más de un valor específico, se trata de un sufijo polisémico.

j. Que 16 de nuestros sufijos son polisémicos; se trata de los sufijos de agente -/'end/-, -/' $\mathrm{er}_{1} /-, \quad-/{ }^{\prime} \mathrm{id}_{2} /-, \quad-/{ }^{\prime}$ ist $_{1} /-, \quad-/{ }^{\prime} \mathrm{i} \beta /-$, $-/{ }^{\prime}$ orj $_{1} /-,-/{ }^{\prime} \mathrm{os}_{2} /-,-/ \mathrm{il} /,-/ \mathrm{V}^{-} \operatorname{der}_{1} /-,-/ \mathrm{V}^{\prime} \mathrm{di} \theta_{1} /-$ y $-/ \mathrm{Vn}^{\prime} \mathrm{der} /-; \mathrm{de}$ los sufijos de relación -/'en $/_{1}{ }_{1}$ e e -/im/ $/ 1_{1}^{-}$; de los sufijos de identificación -l' $\mathrm{er}_{2} /-\mathrm{e}-/$ 'ital y del sufijo de posesor $-/$ 'ent $/$ -

k. Que siete de estos sufijos polisémicos $\left(-/{ }^{\prime} \mathrm{end} /-,-/ \mathrm{er}_{1} /-\right.$, $-/ \mathrm{er}_{2} /-, \quad-/$ ita/, -/'i $\beta /-,-/ \mathrm{im} /{ }_{1}^{-}$, y $\left.-/ \mathrm{V}^{\prime} \mathrm{der}_{1} /-\right)$ no vehiculan ningún valor específico como valor principal.

l. Que nueve de los sufijos no polisémicos $\left(-/^{\prime} \mathrm{an} / 2^{-} \mathrm{e}\right.$ $-/$ 'ika/, de agente; -/'eng/-, -/'in/ y -/ak/-, de relación; -/'i $\mathrm{i} \theta /-\mathrm{y}$ $-j^{\prime}$ ot $/ 2^{-}$, de posesor $y$, en fin, $-l^{\prime}$ ank $/-$ e $-l^{\prime} \mathrm{i} /-$, de hiponimia) presentan una dispersión semántica tal que su valor específico característico se aplica a menos de $51 \%$ de los sufijados que forman.

$m$. Que el sistema de los sufijos formadores de adjetivos en español moderno parece pues presentarse como un sistema poco económico, ya que 125 sufijos son utilizados para vehicular 21 valores; sin embargo, antes de pronunciarse definitivamente so- 
bre la cuestión, será necesario completar ciertos estudios más detallados sobre el uso y la distribución diatópica y diastrática de cada uno de estos sufijos.

\author{
Silvia Faitelson-Weiser \\ Université Laval, Québec
}

\title{
BIBLIOGRAFÍA*
}

*Academia Colombiana de Lexicografía 1975. Breve diccionario de colombianismos. Bogotá.

*Aguilera Patiño, L. 1951. "Diccionario de panameñismos", BAAL, 405-506.

Alarcos Llorach, E. 1969. Gramática estructural. Gredos, Madrid.

Alemany Bolufer, J. 1920. Tratado de la formación de palabras en la lengua castellana. La derivación y la composición. Estudio de los sufijos y prefijos empleados en una y otra. Victoriano Suárez, Madrid.

Alonso, A. 1961. "Noción, emoción, acción y fantasía en los diminutivos", en Estudios lingüisticos, temas españoles. Gredos, Madrid, pp. 161-189.

*Cáceres Freyre, J. 1961. Diccionario de regionalismos de la Provincia de la Rioja. Instituto Nacional de Investigaciones Folklóricas, Buenos Aires.

*Cela, C. J. 1968. Diccionario secreto. Alfaguara, Madrid-Barcelona.

Gorbin, D. 1987. Morphologie dérivationelle et structuration du lexique. Tübingen, 2 ts.

*Cordero Palacios, A. 1957. Léxico de vulgarismos azuayos. Cuenca.

Griado de VAl, M. 1958. Gramática española. Gredos, Madrid.

${ }^{*}$ Doty, E. A. 1958. A glossary of the filipinismos in the Spanish language found in the publications of the period 1890-1920. Michigan [thesis].

FaITELSON-WEISER, S. 1980.Les suffixes quantificateurs de l'espagnol moderne. Éds. Hispaniques, Paris.

— 1981. "Las funciones sufijales en español moderno", en Langues et linguistique. Université Laval, Québec, pp. 95-122. Reproducido en TraLili, 46 (1982), 299-317.

- 1988. DIASLE: Dictionnaire inverse et analyse statistique de la langue espagnole / Diccionario inverso y análisis estadístico de la lengua española / Reverse dictionary and statistical analysis of the Spanish language. Presses de 1' Université Laval, Québec (contiene $1800 \mathrm{pp}$. en forma de microfichas).

*Fernández Naranjo, N., y D. Gómez de Fernández 1967. Diccionario de bolivianismos (con un suplemento de ocho apéndices). Universidad Mayor de San Andrés, La Paz.

${ }^{*}$ Galván, R. A., \& R. V. Teschner 1975. El diccionario del español de Tejas. The dictionary of the Spanish of Texas (Spanish-English). Institute of Modern Language, Silver Spring.

* El asterisco que precede a ciertos títulos indica que se trata de una fuente lexicográfica del DIASLE. 
García De Diego, V. 1951. Gramática histórica española. Gredos, Madrid.

GoOch, A. 1970. Diminutive, augmentative and pejorative suffixes in modern Spanish (A guide to their use and meaning). Pergamon Press, Oxford.

Hanssen, F. 1966. Gramática histórica de la lengua castellana. Eds. HispanoAmericanas, Paris.

* Larousse 1978. Dictionnaire moderne français-espagnol / espagnol-français.

Lathrop, T. A. 1984. Curso de gramática histórica española. Barcelona.

* Lugo, S. DE 1946. Colección de voces y frases provinciales de Canarias. Universidad de Gran Laguna, La Laguna.

Lyons, J. L. 1978. Eléments de sémantique. Paris.

Marcos Marín, F. 1972. Aproximación a la gramática española. Cincel, Madrid.

MEnÉndez PIDAL, R. 1962. Manual de gramática histórica española. EspasaCalpe, Madrid.

Pottier, B. 1953. "Los infijos modificadores en portugués", Boletim de Filologia, 14, 233-256.

1962. Systématique des éléments de relation. Klincksieck, Paris.

Quesada, N. 1935. Lecciones de gramática castellana. Imprenta Española Soley \& Valverde, Madrid.

* Real Academia Española 1970. Diccionario de la Real Academia Española (teniendo en cuenta las correcciones y adiciones de 1967-1980).

— 1973. Esbozo de una nueva gramática de la lengua española. Espasa-Calpe, Madrid.

*Rodríguez Herrera, E. 1958-1959. Léxico mayor de Cuba. Lex, La Habana, 2 ts.

*Santamaría, A., A. Cuartas, y J. Mangada 1975. Diccionario de incorrecciones; particularidades y curiosidades del lenguaje. Paraninfo, Madrid.

* Santamaría, F. J. 1942. Diccionario general de americanismos. P. Robredo, México.

Seco, M. 1972. Gramática esencial del español. Aguilar, Madrid.

Seco, R. 1962. Manual de gramática española. Aguilar, Madrid.

*SolÁ, J. Y. 1950. Diccionario de regionalismos de Salta. Buenos Aires.

TOGEBY, K. 1965. Structure imanente de la langue française. Paris.

*Tovar y Ramírez, E. D. 1966. Vocabulario del oriente peruano. Lima. 


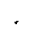

